\title{
Asynchronous parallel disassembly sequence planning for multi-manipulator based on improved shuffled frog leaping algorithm
}

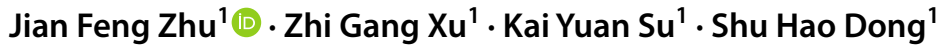

Received: 23 November 2019 / Accepted: 2 April 2020 / Published online: 15 April 2020

(c) Springer Nature Switzerland AG 2020

\begin{abstract}
To solve the problem of low disassembly efficiency of complex products, an asynchronous parallel disassembly sequence planning method of multi-manipulator based on improved shuffled frog leaping algorithm was proposed. Depending on the characteristics of this method, the disassembly manipulators are taken as the research object, and the minimum completion time of disassembly is taken as the optimization objective. Based on the basic disassembly time of parts, the disassembly preparation time caused by the change of disassembly tools and directions is considered, so that the objective function evaluation model is more realistic. Then, the mathematical model of asynchronous parallel disassembly sequence planning is constructed and the disassembly information modeling method is optimized. We proposed a new method based on the fastener constraint matrix and the part constraint matrix to build the information model of product disassembly. Based on this model, the detachable conditions are derived and the feasible disassembly sequence is obtained. In order to improve the performance of the proposed algorithm, a double-link encoding method and an efficient decoding operator is proposed. The adjustment orders which based on adjustment position and the substitution formulas are constructed to carry out the new segmental local evolution of frog individuals, and the update formula of frog position is reconstructed. Furthermore, the two-point mutation operator is added to the global information exchange strategy to enhance the search ability of the algorithm. The proposed algorithm is applied to a screw lifting mechanism and four products of varying complexity, and the results are compared with two algorithms. The results show that the proposed method can solve disassembly problems of different scales and is especially effective in solving large-scale disassembly problems.
\end{abstract}

Keywords Asynchronous parallel disassembly - Disassembly modeling · Improved shuffled frog leaping algorithm . Disassembly sequence planning · Disassembly solution

\section{Introduction}

With the increasingly serious problems of resources and environment, green manufacturing has received extensive attention from academia and industry. Remanufacturing, as a typical form of green manufacturing, uses advanced technology or means to repair and transform waste products so that the quality of the transformed products can reach or exceed new products, thereby achieving the recycling of waste resources [1]. Disassembly is a key process of remanufacturing. It is not only an essential means to achieve an efficient recycling strategy, but also a key step to ensure the quality of remanufacturing products [2]. A good method of Disassembly Sequence Planning (DSP) can effectively improve disassembly efficiency and reduce the disassembly cost [3-6].

Zhi Gang Xu, zhgxu@sdu.edu.cn; Jian Feng Zhu, 775720916@qq.com | ${ }^{1}$ The School of Mechanical Engineering, Shandong University, Jinan 250061, Shandong, China. 
DSP can be divided into two types: sequential disassembly sequence planning (sDSP) and parallel disassembly sequence planning (pDSP) $[7,8]$. The disassembly process of large and complex mechanical products usually has the characteristics of multiple participants, numerous parts and complex constraints, so the research of pDSP is of great significance to the actual disassembly work. According to the execution mode of the task, the pDSP can be divided into synchronous parallel disassembly sequence planning (spDSP) and asynchronous parallel disassembly sequence planning (apDSP). The start time of the disassembly task for each manipulator in spDSP is synchronized. The apDSP allows each manipulator to immediately begin performing their own disassembly tasks if the disassembly priority constraint is met [9]. The solution process of apDSP is more complex than the solution process of spDSP. However, when disassembling complex products, apDSP can reduce the disassembly time and has practical guiding significance.

At present, there are relatively few studies with apDSP. Only the basic disassembly time of the part is considered in the sequence evaluation, which does not meet the actual disassembly. In addition, many algorithms will generate a large number of invalid solutions during the solution process, so that the solution efficiency is low. In view of the above problems and difficulties, we have proposed a novel algorithm for solving apDSP. In the solution process, we will fully consider multi-manipulator collaboration and scheduling, and ensure the feasibility of the solutions in each step of the algorithm. At the same time, during the disassembly process, we will consider the disassembly preparation time caused by the change of disassembly direction and disassembly tool to make the disassembly evaluation more realistic.

The rest of this paper is organized as follows: Sect. 2 reviews the literature on DSP. Section 3 describes the disassembly information model and a mathematical model of apDSP. Section 4 proposes an efficient algorithm based on improved shuffled frog leaping algorithm (ISFLA) to solve the problem of apDSP. Section 5 shows the experimental results of the proposed approach using three study cases. Finally, Sect. 6 concludes our work.

\section{Literature review}

Based on the information model of product disassembly, DSP is aiming at optimizing the given evaluation function of the disassembly sequence and determining the optimal or approximately optimal disassembly sequence that meets the disassembly constraints [10]. DSP is a NP-hard problem [11], many heuristic or metaheuristic methods have been used to solve DSP problems because of the advantages of fast solution speed, convenient parameter setting and the high modularity, such as the, a method to solve DSP which based on genetic algorithm (GA) [12-15], ant colony algorithm $[16,17]$, particle swarm algorithm (PSO) $[18,19]$, artificial bee colony algorithm $[20,21]$, flower pollination algorithm [22], etc.

All the above studies are to solve SDSP, much less work has been done on pDSP. Zhang [23] constructed a disassembly hybrid graph model (DHGM) to describe constraint relationships of product components and got a solution based on the branch-and-bound algorithm, but the solution time will increase dramatically as the scale of the disassembly increases. Tian [24] obtained optimal parallel sequences by constructing chromosome coding structure of parallel sequences and improving crossover and mutation operators of GA. However, a large number of invalid sequences would be generated in the solution process, which would affect the solution efficiency. Cai [25] used the stratified graph to express the parts assembly relationship and proposed a phased iteration strategy based on the ant colony algorithm to solve pDSP problems, however, it is assumed that the basic disassembly time of all components is equal. Zhang [7] proposed a pDSP approach based on fuzzy-rough sets, but this method may produce a sequence that does not meet the disassembly constraint. Ren introduced exclusive and cooperative relationships based on precedence relationships, and optimized the artificial bee colony algorithm to solve the problem of pDSP.

By analyzing the literature on pDSP in the previous paragraph, we can get that they all focus on spDSP, which requires that all disassembly tasks must start simultaneously. In reality, it is unnecessary. The manipulator should be able to perform its next disassembly task immediately if the relevant disassembly constraints are met. However, there is only a very few of literature has studied apDSP, Smith [26] used modular design theory to group parts into modules, created a novel method of DSP for green design, but it was complicated to use modular theory to group parts when the disassembly scale is large. Ren [27] designed an encoding and decoding strategy and solved apDSP by the improving genetic algorithm, but the crossover operation process under the premise of meeting the disassembly constraints is more complicated and only the basic disassembly time of the components is used as the evaluation index.

In summary, although the above methods have achieved significant results in the pDSP, there are still some problems: (1) It does not fully consider multimanipulator collaboration and scheduling on the disassembly process, (2) The current researches are mainly based on SpDSP, and there are relatively few studies on apDSP, (3) many algorithms are prone to generate a large 
number of invalid solutions, affecting the efficiency of the solution, (4) the disassembly model does not express the disassembled components of manipulator at any time, (5) The disassembly evaluation function does not adequately reflect on the actual disassembly process.

In addition, the shuffled frog leaping algorithm(SFLA) [28] is a sub-heuristic collaborative searching swarm intelligent algorithm, which is combined with memetic algorithm(MA) based on genetic genes and particle swarm optimization(PSO) based on the feeding behaviors of particle swarm. It has the characteristics of fast calculation speed, excellent global optimization ability, and strong robustness. The algorithm has been successfully applied to the combinatorial optimization problem of the traveling salesman $[29,30], 0 / 1$ knapsack [31, 32], timetable [33] and Shop Scheduling [34-36]. The algorithm has shown excellent performance. However, no scholars in the literature have applied SFLA to solve DSP problems. Therefore, it is necessary to explore the performance of SFLA in solving DSP problems. In this paper, an improved SFLA is proposed to solve the apDSP problem, which not only ensures the precedence constraints but also takes the disassembly task assignment, human scheduling, asynchronous operations, and sequence evaluation into account.

\section{Problem statement}

To solve the problem of apDSP, we first set up the disassembly information model which represents disassembly constraint through the fastener constraint matrix $G_{1}$ and the part constraint matrix $G_{2}$, then, we set up the mathematical model of apDSP.

\subsection{Construction of disassembly information model}

The disassembly information model is used to represent disassembly constraints and generate a feasible disassembly sequence. The precedence relationships between components must be strictly followed when solving DSP problems. To depict precedence relations, we supplement and optimize Gonzalez's [37] modeling method and set up a model based on the fastener constraint matrix and the part constraint matrix.

We make some explanation for the product disassembly modeling: the part refers to the minimum disassembly unit except the fastener. Fasteners are mechanical parts used for fastening two or more parts, such as screws, bolts, studs, etc. Components are the collective name for parts and fasteners.

\subsubsection{Fastener constraint matrix $\left(G_{1}\right)$}

It represents the constraint relationship between the fastener and the part of the disassembly product.

$G_{1}=\left\{c O_{i j}\right\}_{m \times n}=\left[\begin{array}{cccc}c O_{11} & c O_{12} & \cdots & c O_{1 n} \\ c O_{21} & c O_{22} & \cdots & c O_{2 n} \\ \vdots & \vdots & & \vdots \\ & & & \\ c O_{m 1} & c O_{m 2} & \cdots & c O_{m n}\end{array}\right]$

$i$ : the index of fastener, $i \in\{1,2, \ldots m\}$, where $m$ is the number of all fasteners in disassembly model; $j$ : the index of part,$j \in\{1,2, \ldots n\}$, where $n$ is the number of all parts in disassembly model.

$c o_{i j}=\left\{\begin{array}{lll}0, & \text { if } & \text { fastener } i \text { has no constraint relationship with part } j \\ 1, & \text { if } & \text { fastener } i \text { fastens part } j \\ 2, & \text { if } & \text { fastener } i \text { is blocked by part } j\end{array}\right.$

\subsubsection{Part constraint matrix $\left(G_{2}\right)$}

It represents the constraint relationship between different parts of the disassembly product.

$$
\begin{aligned}
& G_{2}=\left\{r e_{i j}\right\}_{n \times n}=\left[\begin{array}{cccc}
r e_{11} & r e_{12} & \ldots & r e_{1 n} \\
r e_{21} & r e_{22} & \cdots & r e_{2 n} \\
\vdots & \vdots & & \vdots \\
r e_{n 1} & r e_{n 2} & \ldots & r e_{n n}
\end{array}\right]
\end{aligned}
$$

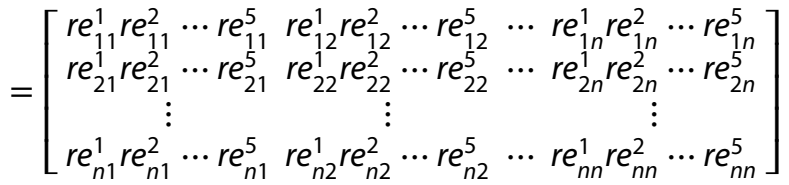

$i$, $j$ : both the index of the part, $i, j \in\{1,2, \ldots n\}$, where $n$ is the number of all parts in disassembly model

$r e_{i j}^{d}=\left\{\begin{array}{ll}0, & \text { part } i \text { has no interference with part jin direction } d \\ 1, & \text { part } i \text { is interfered by part } j \text { in direction } d\end{array}\right.$,

$d$ : the index of disassembly directions, $i \in\{1,2, \ldots s\}$, where $s$ is the number of all disassembly directions.

Through $G_{1}$ and $G_{2}$, we can fully express the disassembly model information. In this modeling approach, the conditions that fastener $i$ can be disassembled are as follows:

$$
\left\{\begin{array}{l}
\sum_{j=1}^{j=n} \operatorname{Judge}\left(c o_{i j}\right)=0 \\
\operatorname{Judge}\left(c o_{i j}\right)= \begin{cases}0, & c o_{i j} \neq 2 \\
1, & c o_{i j}=2\end{cases}
\end{array}\right.
$$


The condition for the disassembly of part $i$ without relevant fastener fixation is as follows:

$\prod_{d=1}^{s}\left(\sum_{j=1}^{n} r e_{i j}^{d}\right)=0$

One disassembly product showed in Fig. 1, it is assembled with 6 fasteners and 4 parts. Since it is a two-dimensional assembly drawing and the bottom of the product is fixed, only 3 disassembly directions are considered: $+x$, $-x,+y$. According to the above modeling method, the fastener constraint matrix $G_{1}$ and the part constraint matrix $G_{2}$ of the product can be obtained as follows:

$G_{1}=\left[\begin{array}{llll}1 & 0 & 1 & 0 \\ 1 & 1 & 2 & 0 \\ 0 & 1 & 1 & 0 \\ 1 & 1 & 0 & 2 \\ 0 & 1 & 0 & 1 \\ 1 & 0 & 0 & 1\end{array}\right]$

$G_{2}=\left[\begin{array}{llll}0 & 1 & 5 & 3 \\ 0 & 0 & 5 & 3 \\ 2 & 2 & 0 & 0 \\ 4 & 4 & 0 & 0\end{array}\right]=\left[\begin{array}{llll}000 & 001 & 101 & 011 \\ 000 & 000 & 101 & 011 \\ 010 & 010 & 000 & 000 \\ 100 & 100 & 000 & 000\end{array}\right]$.

\subsection{The construction method of feasible disassembly sequences}

The feasible disassembly sequence refers to the disassembly sequence that satisfies the disassembly constraint relationship. The steps for generating a feasible disassembly sequence according to the above disassembly information modeling method are as follows:

1. Define $A$ as the set of disassembly components, $X$ is one feasible disassembly sequence, make $A=\emptyset, X=\emptyset$;
2. In the initial state, according to matrix $G_{1}$ and matrix $G_{2}$ search for all disassembly components and put them into $A$, define temporary transformation matrix $G_{3}$ and $G_{4}$, make $G_{3}=G_{1}, G_{4}=G_{2}$;

3. Randomly select an element $a$ from $A$, put it into the left-most end of $X$ and delete element $a$ in $A$; then judge the type of element $a$, if element $a$ is a fastener, go to step4, if element $a$ is a part, then go to step5;

4. Update matrix $G_{3}$ (delete all the elements in row of $a$ in $G_{3}$ ). According to matrix $G_{1}$, for each part connected by fastener $a$, judge whether these parts can be disassembled according to formula (4). If it is, put the index of this part into $A$; if not, go to step6;

5. Update $G_{3}$ (delete all the elements in line of $a$ in $G_{3}$ ) and update $G_{4}$ (delete all the elements in column of $a$ in $G_{4}$ ). According to $G_{1}$, for each fastener blocked by part $a$, judge whether it is can be disassembled according to formula (3). If it is, put the index of the corresponding fastener into $A$. In addition, according to $G_{2}$, for each part blocked by part $a$ in any disassembly direction, judge whether it can be disassembled according to formula (4).If it can be disassembled and the part is not in $X$, put the index of the corresponding part into $A$;

6. Determine if all fasteners and parts in the product have been disassembled. If it completes, output $X$, otherwise go to step3.

\subsection{Mathematical model}

The notations in model are summarized as follows:

1. $p_{i}$-the index of a component, $i \in\{1,2, \ldots, N\}$ ,$N=n+m$, where $n$ is the number of all parts in disassembly product, and $m$ is the number of all fasteners.

2. $m_{k}$-the index of a manipulator, $k \in\{1,2, \ldots l\}$, where $l$ is the number of all manipulators.

3. $x_{k}^{j}$-the index of a disassembly task, it represent the $j$ th disassembly task of manipulator $m_{k}, j \in\left\{1,2, \ldots Q_{k}\right\}$,
Fig. 1 An example of a product to be disassemble

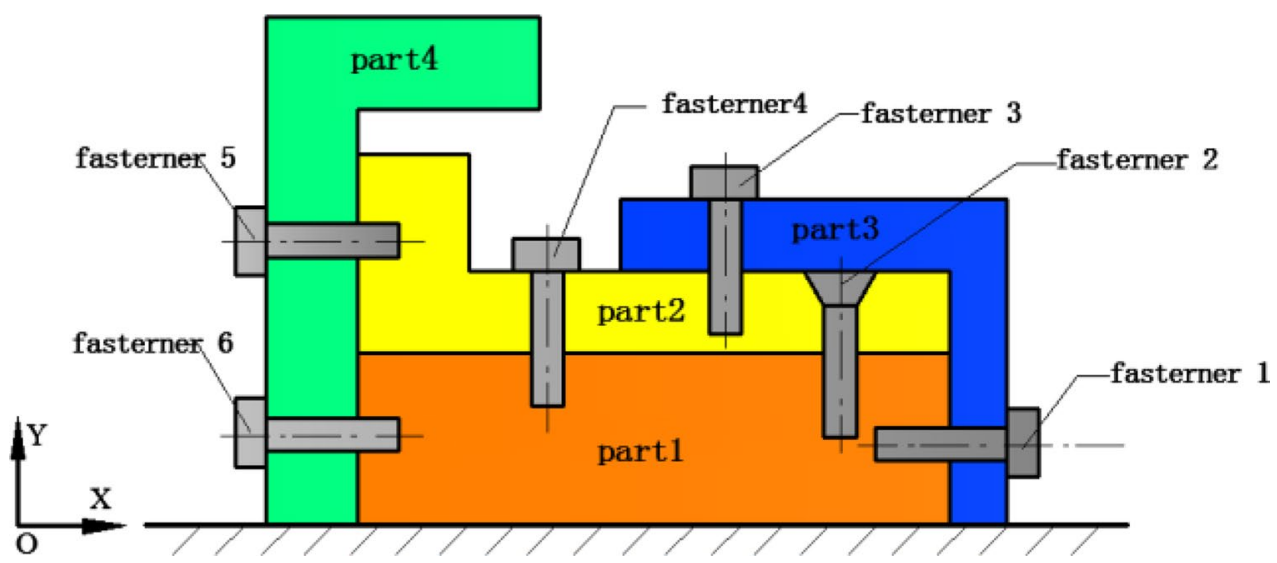


where $Q_{k}$ is the number of all disassembly task for manipulator $m_{k}$.

4. $s t_{k}^{j}$-the start disassembly time for the jth disassembly task of manipulator $m_{k}$.

5. $b t_{k}^{j}$-the basic disassembly time for the jth disassembly task of manipulator $m_{k}$.

6. $r t_{k}^{j}$-the preparation time before the $j$ th disassembly task of manipulator $m_{k}$.

7. $e t_{k}^{j}$-the completion time for the $j$ th disassembly task of manipulator $m_{k}$.

8. $n f_{k}$-the index of the disassembly task currently being executed by manipulator $m_{k}$

9. $c p_{k}^{j}$-the set of feasible disassembly tasks after $m_{k}$ completes the jth disassembly task.

In order to efficiently disassemble all components, we aim to minimize the disassembly completion time and determine the optimal feasible disassembly sequence that satisfies the following constraints:

1. Meet precedence constraints;

2. All components are disassembled and can only be disassembled once;

3. Each manipulator must execute at least one disassembly task;

4. The manipulator can only disassemble one part at a time.

In fact, it is to determine the optimal disassembly task allocation plan for all manipulators under the condition of comprehensively considering disassembly constraints and disassembly resources. We make the following assumptions:

1. There is no interference in the working area of manipulators;

2. The basic disassembly time for disassembling the same components by different manipulators is the same.

Based on the above-mentioned modeling method and notation description, a mathematical model of apDSP is formulated as follows:

$$
\begin{aligned}
& \operatorname{Min}\left\{\max \left(e t_{1}^{Q_{1}}, e t_{2}^{Q_{2}}, \ldots, e t_{k}^{Q_{k}} \ldots, e t_{l}^{Q_{1}}\right)\right\} \\
& \sum_{k=1}^{1} \sum_{j=1}^{Q_{k}} x_{k}^{j}=\sum_{i=1}^{N} p_{i} \\
& \sum_{j=1}^{Q_{k}} x_{k}^{j} \geq 1
\end{aligned}
$$

$$
\begin{aligned}
& \bigcap_{k=1}^{l} \sum_{j=1}^{Q_{k}} x_{k}^{j}=\emptyset \\
& \mathrm{et}_{\mathrm{k}}^{j}=s t_{\mathrm{k}}^{j}+b \mathrm{t}_{\mathrm{k}^{\prime}}^{j} 1 \leq j \leq Q_{k^{\prime}}, \forall k \\
& \left\{\begin{array}{l}
s t_{k}^{j} \geq \mathrm{et}_{\mathrm{k}}^{j-1}+r t_{\mathrm{k}}^{j} \\
s t_{\mathrm{k}}^{j}=e t_{\mathrm{k}}^{j-1}+r t_{\mathrm{k}^{\prime}}^{j}
\end{array} i x_{k}^{j} \in c p_{k}^{j}, \quad 2 \leq j \leq Q_{k^{\prime}}, \forall k\right.
\end{aligned}
$$

$r t_{k}^{j}=r_{1} t_{k}^{j}+r_{2} t_{k^{\prime}}^{j} 2 \leq j \leq Q_{k^{\prime}} \quad \forall k$

$\bigcap_{j=1}^{Q_{k}} c p_{k}^{j}=\emptyset, \forall k$

Constraint (5) addresses the optimization goal is to minimize the disassembly completion time; Constraint (6) addresses all components must be disassembled; Constraint (7) addresses each manipulator must execute the disassembly task at least once; Constraint (8) addresses that each component can only be disassembled once, there is no intersection of the disassembly tasks for each manipulator; Constraint (9), (10) and (11) address the relationship between $t$ start disassembly time, basic disassembly time, preparation time, and completion time. In constraint (12), $r_{1} t_{k}^{j}$ represents the preparation time added by the replacement of the disassembly tool, and $r_{2} t_{k}^{j}$ represents the preparation time due to the change of the disassembly direction. We define it as follows:

$r_{1} t_{k}^{j}= \begin{cases}1, & \begin{array}{l}\text { if } m_{k} \text { needs to change the disassembly tool } \\ \text { when executinging the disassembly task } x_{k}^{j}\end{array} \\ 0, & \begin{array}{l}\text { if } m_{k} \text { does not needs to change the disassembly tool } \\ \text { when executinging the disassembly task } x_{k}^{j}\end{array}\end{cases}$

$$
r_{2} t_{k}^{j}=\left\{\begin{array}{ll}
1, & \text { if } m_{k} \text { needs to change the disassembly direction } \\
\text { when executinging the disassembly task } x_{k}^{j} \\
0, & \text { if } m_{k} \text { does not needs to change the disassembly direction } \\
\text { when executinging the disassembly task } x_{k}^{j}
\end{array} .\right.
$$

\section{The proposed approach}

This section presents an improved SFLA to solve the apDSP problem. The main steps of the proposed approach are shown as follows:

1. Solution encoding and population initialization;

2. Decoding approach 
3. Evaluation of objective functions;

4. Frog grouping method

5. Local evolution method of frog groups

6. Strategy of global information interaction

7. Termination rule

\subsection{Solution encoding and population initialization}

The encoding structure of the frog corresponds to the solution of the apDSP problem, each frog individual is represented as a feasible parallel disassembly sequence. The following definitions are made for the encoding structure of frogs:

$$
\begin{aligned}
\text { Frog } & =\left\{\left\{f_{p}\right\}_{1 \times n} \mid\left\{f_{m}\right\}_{1 \times n}\right\} \\
& =\left\{p_{1}, p_{2}, \ldots, p_{i} \ldots, p_{N} \mid m_{1}, m_{2}, \ldots, m_{k} \ldots, m_{l}\right\}
\end{aligned}
$$

We design a double-link encoding structure that includes disassembly task sequence $f_{p}$ and the disassembly manipulator sequence $f_{m}$, where both the length of $f_{p}$ and $f_{m}$ are $N$. The elements of the same position in the two sequences are one-to-one correspondence. It indicates that the disassembly task of the position is executed by the corresponding manipulator.

$\mathrm{F}$ o $\mathrm{r}$

i $n \quad s \quad t \quad a \quad n \quad c$, Frog $=\{4,5,3,1,2,6,8,7,9 \mid 3,1,1,2,3,1,3,2,2\}$. The sequence represents 9 disassembly tasks are performed by 3 manipulators, so that we can get the disassembly task of each manipulator:

$x_{1}^{1}=5, x_{1}^{2}=3, \quad x_{1}^{3}=6 ; \quad x_{2}^{1}=1, x_{2}^{2}=7, x_{2}^{3}=9 ;$

$x_{3}^{1}=4, x_{3}^{2}=2, x_{3}^{3}=8$

In addition, $f_{p}$ is a feasible disassembly sequence generated based on the disassembly information model; $f_{m}$ is a randomly generated disassembler sequence, and the frog's encoding structure ensures that each frog in the initial population is a feasible sequence, which helps to improve search efficiency. The generation of a feasible individual Frog is presented as follows:

Step 1 Set $N$ (the total number of disassembly tasks) and I (the total number of manipulators),

Step 2 Construct a feasible disassembly task sequence according to the method described in Sect. 3.2,

Step 3 According to the number of manipulators, randomly generated $f_{m}$.

\subsection{Decoding approach}

In this paper, our optimization goal is to minimize the disassembly completion time. In fact, the apDSP is a scheduling scheme for the start disassembly time and the end disassembly time of each disassembly task for each manipulator, so it is necessary to decode individual into the timing of each disassembly task. Further, under the condition of precedence constraint, we perform parallel scheduling of the disassembly time of each disassembly task. In view of the individual coding mode and the characteristics of apDSP, we propose the frog individual decoding operator. The specific steps are as follows:

1. According to the individual code, determine the disassembly task $x_{k}^{j}$ of each manipulator $m_{k^{\prime}}$, initialize $s t_{k^{\prime}}^{j} b t_{k^{\prime}}^{j}$ $r t_{k^{\prime}}^{j} e t_{k^{\prime}}^{j} n f_{k^{\prime}} c c_{k^{\prime}}^{j^{\prime}}$ generate a component set vP that can be disassem bled in an initial state based on $G_{1}$ and $G_{2}$;

2. For the first disassembly task $x_{k}^{1}$ of each $m_{k}$, judge whether it belongs to $v P$; if it belongs, set $s t_{k}^{1}=0$, update $e t_{k^{\prime}}^{1}$ according to formula (9), and update $c p_{k}^{1}$ and $n f_{k i}$

3. Judge the current disassembly task $x_{k}^{n f_{k}}$ of each $m_{k}$ in turn, if $x_{k}^{n f_{k}} \in\left[v P \cup\left(\sum_{j=1}^{n f_{k}-1} c p_{k}^{j}\right)\right]$, update $s t_{k}^{n f_{k}}, e_{k}^{n f_{k}}$ based on formulas (9), (10) and (11), also update $c p_{k}^{n f_{k}}$ and $n f_{k}$; otherwise go to step4;

4. For the current disassembly task $x_{k}^{n f_{k}}$, judge whether it belongs to other manipulator's set $c p_{k_{-} \text {other }}$ if only one set includes $x_{k}^{n f_{k}}$, determine the position of $x_{k}^{n f_{k}}$ in $c p_{k_{\text {other }}}$. Assume that the location is $c p_{k_{-} \text {other }}^{\text {pos }}$ if

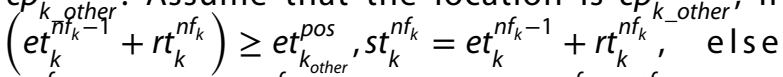
$s t_{k}^{n f_{k}}=e t_{k_{-} \text {other }}^{\text {pos }}+r t_{k}^{n f_{k}}$, and also update e $t_{k}^{n f_{k}}, c p_{k}^{n f_{k}}$ and $n f_{k}$. otherwise go to step5;

5. If $x_{k}^{n f_{k}}$ in many sets, determine the position of $x_{k}^{n f_{k}}$ in there sets, assume the location set is $\left\{\quad c p_{k_{-} \text {other 1 }}^{\text {pos }_{1}} c p_{k_{-} \text {other2 } 2}^{\text {pos }_{2}}, \ldots, c p_{k_{-} \text {otheri }}^{\text {pos }_{1}}\right\}, \mathrm{s}$ e $\mathrm{t}$

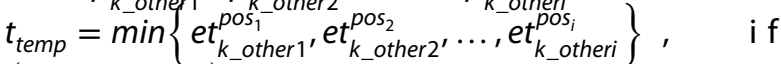
$\left(e t_{k}^{n f_{k}-1}+r t_{k}^{n f_{k}}\right) \geq t_{\text {temp }}, s t_{k}^{n f_{k}}=e t_{k}^{n f_{k}-1}+r t_{k}^{n f_{k}}$, else $s t_{k}^{n f_{k}}=t_{\text {temp }}+r t_{k}^{n f_{k}}$, and also update et $t_{k}^{n f_{k}}, c p_{k}^{n f_{k}}$ and $n f_{k}$;

6. Judge whether all disassembly task has been scheduled. If yes, output of the scheduling plan. Otherwise, return to step 3 .

Take the product to be disassembled in Fig. 1 as an example, fix the part 1, and set the basic disassembly information as follows.

In Table 1, Dir represents disassembly direction; $B t$ represents the basic disassembly time; Next, take a feasible sequence as an example to explain its decoding process in detail.

Set Frog $=\{5,9,7,10,3,4,6,8,2 \mid 2,1,2,1,1,2,2,1,1$,$\} ,$ This sequence represents 9 disassembly tasks are performed by 2 manipulators. It can be obtained from the encoding structure and the disassembly information matrix $G_{1}$ and $G_{2}$ : 
Table 1 Disassembly information of a product

\begin{tabular}{lllll}
\hline Index & Component & Tool & Dir & Bt/s \\
\hline 1 & Part 1 & Handwork T0 & $+Y$ & 0 \\
2 & Part 2 & Handwork T0 & $+Y$ & 2 \\
3 & Part 3 & Handwork T0 & + Y & 4 \\
4 & Part 4 & Handwork T0 & + Y & 3 \\
5 & Fastener 1 & Wrench T1 & + X & 3 \\
6 & Fastener 2 & Screwdriver T2 & + Y & 3 \\
7 & Fastener 3 & Wrench T1 & + Y & 2 \\
8 & Fastener 4 & Wrench T1 & $+Y$ & 2 \\
9 & Fastener 5 & Wrench T1 & $-X$ & 2 \\
10 & Fastener 6 & Wrench T1 & $-\mathrm{X}$ & 2 \\
\hline
\end{tabular}

1. $x_{1}^{1}=9, x_{1}^{2}=10, x_{1}^{3}=3, x_{1}^{4}=8, x_{1}^{5}=2 ; x_{2}^{1}=5, x_{2}^{2}=7$ $, x_{2}^{3}=4, x_{2}^{4}=6 ; v P=\{5,7,9,10\}$

2. Because $x_{1}^{1}, x_{2}^{1} \in v P, s t_{1}^{1}=0, s t_{2}^{1}=0$; then $e t_{1}^{1}=s t_{1}^{1}+b t_{1}^{1}=2, e t_{2}^{1}=3, n f_{1}=2, n f_{2}=2$;

3. As for $x_{1}^{2}$, because $x_{1}^{2} \in v P$ and $r t_{1}^{2}=0$, $s t_{1}^{2}=e t_{1}^{1}+r t_{1}^{2}=2, e t_{1}^{2}=4, n f_{1}=3, c p_{1}^{2}=\{4\}$;

4. As for $x_{2}^{2}$, because $x_{2}^{2} \in v P$ and $r t_{2}^{2}=1$, $s t_{2}^{2}=e t_{2}^{1}+r t_{2}^{2}=4, e t_{2}^{2}=6, n f_{2}=3, c p_{2}^{2}=\{3\}$

5. As for $x_{1}^{3}$, because it does not belong to $v P \cup C p_{1}^{2}$, but $x_{1}^{3} \in c p_{2}^{2}$, because $e t_{1}^{2}+r t_{1}^{3}=6=e t_{2}^{2}$, then $s t_{1}^{3}=6$, $e t_{1}^{3}=10$, and $n f_{1}=4, c p_{1}^{3}=\{8\}$;

6. According to the above method, we can get the disassembly time of the remaining disassembly tasks. The result is shown in Fig. 2.

In Fig. 2, the number of each color block presents the index of disassembly task number, the length of the color block presents the disassembly time, and the shaded portion presents the preparation time. The apDSP is a more complex disassembly problem than spDSP. It eliminates the limitation of SPDP (i.e., the cooperative tasks must begin at the same time). Further, the disassembly time of each part might affect the disassembly process of aPDP. If we use the synchronous disassembly method to disassemble the above example, the result is shown in Fig. 3.

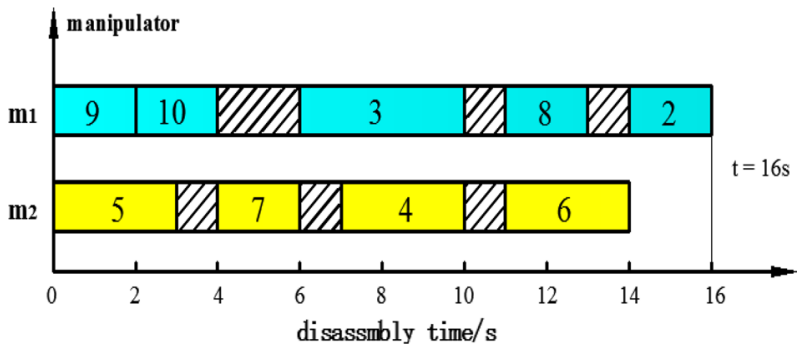

Fig. 2 Asynchronous parallel disassembly planning result
The experimental results show that the decoding method proposed in this paper can effectively shorten the disassembly completion time.

\subsection{Evaluation of objective functions}

In this paper, we use the optimization objective function as the fitness function and define it as follows:

$f i t(F r o g)=\operatorname{Min}\left\{\max \left(e t_{1}^{Q_{1}}, e t_{2}^{Q_{2}}, \ldots, e t_{k}^{Q_{k}}, \ldots, e t_{l}^{Q_{1}}\right)\right\}$

According to the decoding operator, it can be obtained and the fitness function value can be obtained based on the disassembly completion time of each manipulator. The smaller the fitness value, the better the individual quality.

\subsection{Frog grouping method}

After constructing the initial frog group according to the coding structure rules of the frog individual, the fitness of all frog individuals in the population can be calculated according to formula (16), and then arrange all fitness values in ascending order. The grouping formula is as follows:

$Y^{i}=\left\{\operatorname{Frog}_{i+q_{s p}(j-1)} \mid 1 \leq j \leq q_{s f}\right\}, 1 \leq i \leq q_{s p}$

where $Y^{i}$ is the index of frog subpopulations, $q_{s p}$ is the number of frog subpopulations, $q_{s f}$ is the number of frog individuals in each subpopulation $, i, j$ is an integer.

\subsection{Local evolution method of frog groups}

Since the local search strategy of the standard frog leaping algorithm is not suitable for solving the apDSP problem, we introduce the adjustment factor and the adjustment order to optimize the local search strategy. The adjustment order based on the adjustment position is used to adjust the disassembly task sequence, and the double-point replacement method is used to adjust the manipulator sequence. In final, we rebuilt the position update formula of the frog individual.

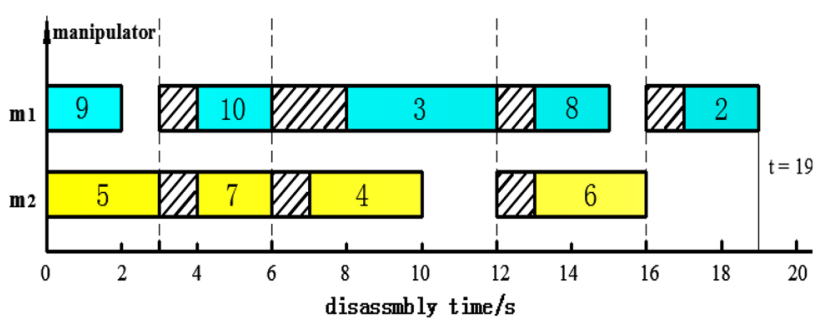

Fig. 3 Synchronous parallel disassembly planning result 


\subsubsection{Adjustment method for disassembly task sequence}

In the solution of the traveling salesman (TSP) problem, the adjustment factor is expressed as changing the order of access of the two cities, and the adjustment order is an ordered sequence composed of multiple adjustment factors. In this paper, based on the apDSP problem, the adjustment order based on the adjustment position is used to adjust the disassembly task sequence.

1. Adjustment factor

$f_{p}=\left\{p_{1}, p_{2}, \ldots, p_{i} \ldots, p_{n}\right\}$, which present a disassembly task sequence; We define the adjustment factor as $A p(\alpha, \beta)$, which is to take the component $\beta$ th in $f_{p}$ insert into the front of the $\alpha$ th component, and generate a new sequence.

For example, $f_{p}=(3,2,1,5,6,4)$, adjustment factor is $A p(3,5)$, so newf $f_{p}=f_{p}+A p(3,5)=(3,2,6,1,5,4)$.

2. Adjustment order based on adjustment position

$\operatorname{Aps}(\gamma, \delta)=\left(A p_{1}, A p_{2}, \ldots, A p_{n}\right)$, it presents an adjustment order and the number of adjustment factors are $n$; where $\gamma, \delta$ presents adjustment position which randomly generated and $1 \leq \gamma \leq \delta \leq n$; where $A p_{1}, A p_{2}, \ldots, A p_{n}$ represent adjustment factor, the order between them is meaningful. The adjustment order acts on the disassembly sequence, which corresponds to all of the adjustment factors in the adjustment order acting in turn on the disassembly sequence.

There are two different disassembly task sequences $f_{p a^{\prime}}$ $f_{p b}$; The formula $\operatorname{Aps}(\gamma, \delta) \otimes\left(f_{p a} \Theta f_{p b}\right)$ represents that the sequence between the component $\gamma$ th and the component $\delta$ th in $f_{p a}$ is adjusted according to the arrangement of the sequence in $f_{p b}$ :

$$
\text { new } \begin{aligned}
f_{p a} & =f_{p a}+A p s(\gamma, \delta) \otimes\left(f_{p a} \Theta f_{p b}\right)=f_{p a}+\left(A p_{1}, A p_{2}, \ldots, A p_{n}\right) \\
& =\left[\left(f_{p a}+A p_{1}\right)+A p_{2}\right]+\cdots+A p_{n}
\end{aligned}
$$

The specific steps of constructing the adjustment order are as follows:

1. Set $i, j$; where $j$ is the position of the component in $f_{p a}$ $\gamma \leq j \leq \delta$; where $i$ is the relative position of this component in $f_{p b}, 1 \leq i \leq \delta-\gamma+1$; let $i=1, j=\gamma$;

2. Judge whether $f_{p b}(i)$ is equal to $f_{p a}(j)$, if $f_{p b}(i) \neq f_{p a}(j)$, then find the component position $v$ in $f_{p a}$ which can make $f_{p b}(i)==f_{p a}(v)$, so that we can get one adjustment factor $A p(j, v)$, newf $p a=f_{p a}+A p(j, v)$; if $f_{p b}(i)=f_{p a}(j)$, go to step3;

3. $i=i+1, j=j+1$; if $i>\delta-\gamma+1$, the adjustment ends, otherwise, go to step 2 and continue.
For example, $f_{p a}=(3,6,1,5,4,2), f_{p b}=(1,6,4,5,2,3)$, based on the above construction method, we can get:

$\operatorname{Aps}(2,5) \otimes\left(f_{p a} \Theta f_{p b}\right)=\left(A p_{1}(2,3), A p_{2}(4,5)\right)$.

\subsubsection{Adjustment method for manipulator sequence}

In this paper, the manipulator sequence $f_{m}$ is adjusted by the method of double-point replacement. The replacement formula is defined as follows:

$A m(\varepsilon, \tau) \otimes\left(f_{m a} \Theta f_{m b}\right)$

where $\varepsilon, \tau$ is a randomly generated replacement position; where $f_{m a}$ is the sequence to be adjusted, and $f_{m b}$ is the reference sequence.

Its role is to replace the element $\varepsilon$ th and element $\tau$ th in $f_{m a}$ with the element where in the corresponding position in $f_{m b}$, so that $f_{m a}(\varepsilon)=f_{m b}(\varepsilon), f_{m a}(\tau)=f_{m b}(\tau)$.

\subsubsection{Update strategy for frog individual}

Assume that the worst individual in the current iteration subgroup is Frog $_{w}$, the best individual is Frog $_{b}$, and the global optimal individual is rrog $_{g}$. The position adjustment strategy of the worst frog individual Frog ${ }_{w}$ is as follows:

$s=\min \left\{\operatorname{int}\left[r \cdot\right.\right.$ length $\left.\left.\left(\operatorname{Aps}(\gamma, \delta) \otimes\left(f_{p w} \Theta f_{p b}\right)\right)\right], s_{\max }\right\}$

$\left\{\begin{array}{l}\operatorname{newf}_{p w}=f_{p w}+\sum_{i=1}^{s}\left(A p_{i}\right) \\ A p_{i} \in \operatorname{Aps}(\gamma, \delta) \otimes\left(f_{p w} \Theta f_{p b}\right)\end{array}\right.$

newf $f_{m w}=A m(\varepsilon, \tau) \otimes\left(f_{m w} \Theta f_{m b}\right)$

where $r$ represents a random number distributed between $[0,1]$; length $\left(\operatorname{Aps}(\gamma, \delta) \otimes\left(f_{p w} \Theta f_{p b}\right)\right)$ represents the length of the adjustment order; int means to convert it to an integer; and $s_{\max }$ represents the max leapfrog step; $\sum_{i=1}^{s}\left(A p_{i}\right)$ represents the adjustment orders of $f_{p w}$. After executing the above update strategies, if fit $\left(\right.$ newFrog $\left._{w}\right)<$ fit $\left(\right.$ Frog $\left._{w}\right)$, update Frog $_{w}$, otherwise, execute the following update strategy:

$s=\min \left\{\operatorname{int}\left[r \cdot\right.\right.$ length $\left.\left.\left(A p s(\gamma, \delta) \otimes\left(f_{p w} \Theta f_{p g}\right)\right)\right], s_{\max }\right\}$

$\left\{\begin{array}{l}\operatorname{newf}_{p w}=f_{p w}+\sum_{i=1}^{s}\left(A p_{i}\right) \\ A p_{i} \in A p s(\gamma, \delta) \otimes\left(f_{p w} \Theta f_{p g}\right)\end{array}\right.$

newf $f_{m w}=A m(\varepsilon, \tau) \otimes\left(f_{m w} \Theta f_{m g}\right)$

Continue to execute the above updating strategy. If the fitness value of Frog $_{w}$ is still not improved, then, reconstruct 


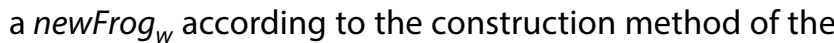
initial frog individual.

\subsection{Strategy of global information interaction}

Because the frog individuals may be trapped in the local optimal solution, the mutation operation is added to enhance the diversity of the population in the process of global information interaction. We use the two-point mutation method to construct the mutation operator. The basic idea is as follows.

Two mutation points are randomly generated in $f_{m}$, the manipulator at the position is mutated and randomly changed to other manipulators.

As mentioned above, the encoding and decoding methods of the frogs mentioned in this paper can ensure the feasibility of the initial solution. At the same time, the frog individuals directly generate feasible solutions after local evolution and global information interaction, which improves the convergence speed of the algorithm.

\subsection{Termination rule}

The termination condition of the algorithm proposed in this paper is the number of population iterations. Flow chart is shown in Fig. 4.

\section{Experimental results and analysis}

This section assesses the performance of the proposed method through several test cases. All experiments are executed in VS2015 and run on an Intel(R) Core(TM) i3-4170 CPU (3.7 GHz/4.00-GB RAM) PC with a Windows 7 operating system.
Fig. 4 Basical flow chart of improved SFLA

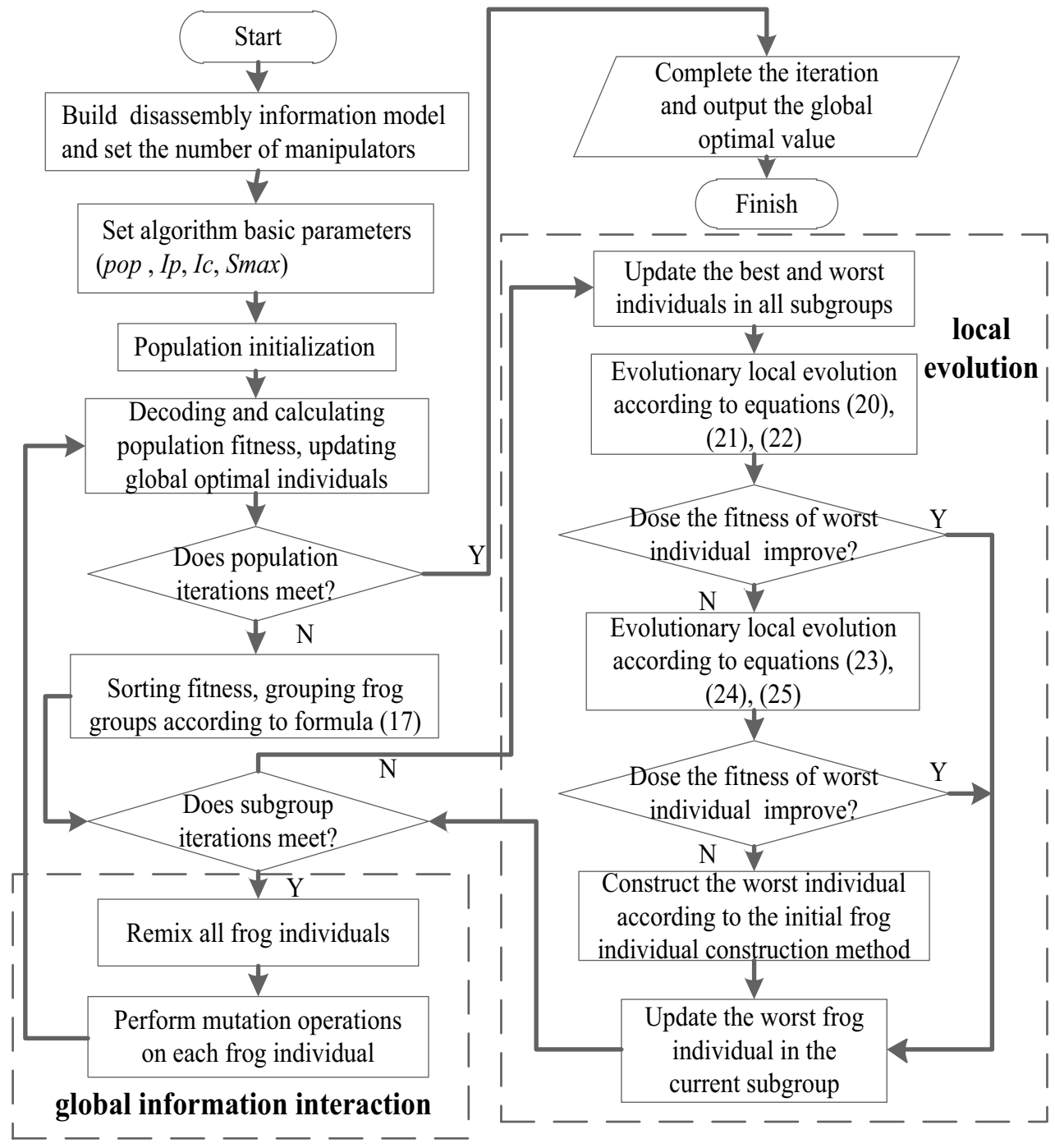




\subsection{Case study 1}

First, the proposed algorithm is verified by the disassembly of one screw lifting mechanism shown in Fig. 5 (including 33 parts and 13 fasteners). The disassembly information about this model is shown in Table 2, further, the fastener constraint matrix $G_{1}$ and the part constraint matrix $G_{2}$ are available (considering the disassembly direction $\pm \mathrm{x}, \mathrm{y} \pm \mathrm{z}$ ).

\begin{tabular}{|c|c|c|}
\hline & 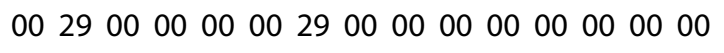 & 000000000000000000000000000000 \\
\hline & 300000012900002900000000000000 & 000000000000000000000000000000000000 \\
\hline & $\begin{array}{llllllllllllll}00 & 02 & 01 & 29 & 00 & 00 & 29 & 00 & 00 & 00 & 00 & 00 & 00 & 00\end{array}$ & $\begin{array}{lllllllllllllllllll}00 & 00 & 00 & 00 & 00 & 00 & 00 & 00 & 00 & 00 & 00 & 00 & 00 & 00 & 00 & 00 & 00\end{array}$ \\
\hline & 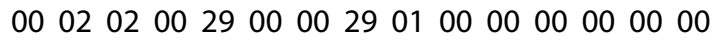 & $\begin{array}{lllllllllllllllllll}00 & 00 & 00 & 00 & 00 & 00 & 00 & 00 & 00 & 00 & 00 & 00 & 00 & 00 & 00 & 00\end{array}$ \\
\hline & 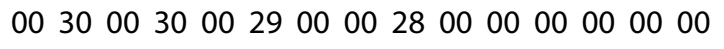 & 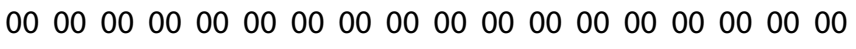 \\
\hline & 003000003000002800000005050516 & 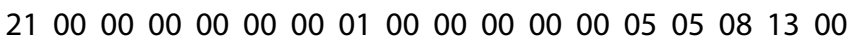 \\
\hline & $\begin{array}{llllllllllllll}30 & 00 & 00 & 00 & 28 & 00 & 30 & 00 & 00 & 00 & 00 & 00 & 00 & 00\end{array}$ & 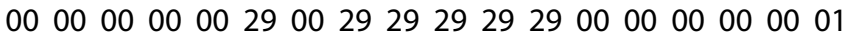 \\
\hline & 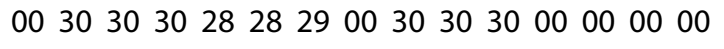 & 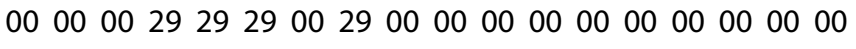 \\
\hline & 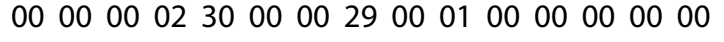 & 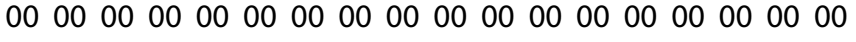 \\
\hline & 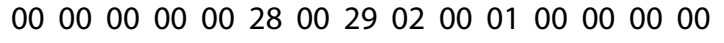 & 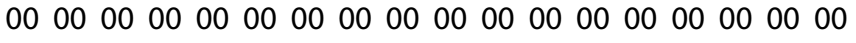 \\
\hline & $\begin{array}{llllllllllllll}00 & 00 & 00 & 30 & 00 & 29 & 00 & 02 & 00 & 00 & 00 & 00 & 00\end{array}$ & $\begin{array}{llllllllllllllllll}00 & 00 & 00 & 00 & 00 & 00 & 29 & 00 & 00 & 00 & 00 & 00 & 00 & 00 & 00 & 00 & 00\end{array}$ \\
\hline & 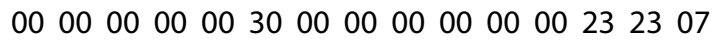 & 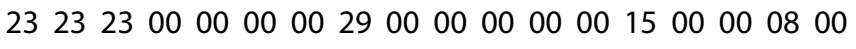 \\
\hline & 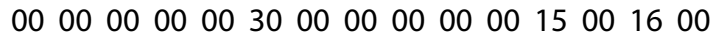 & 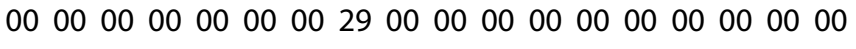 \\
\hline & $\begin{array}{llllllllllllll}00 & 00 & 00 & 00 & 30 & 00 & 00 & 00 & 00 & 00 & 15 & 08 & 00 & 00\end{array}$ & 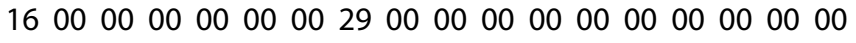 \\
\hline & 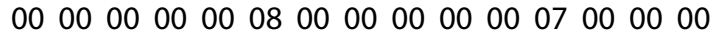 & 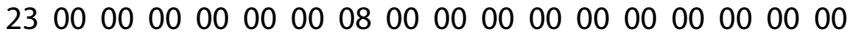 \\
\hline & 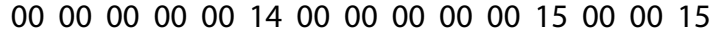 & 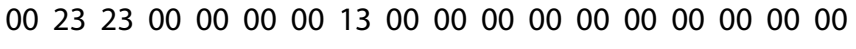 \\
\hline$G_{2}=$ & 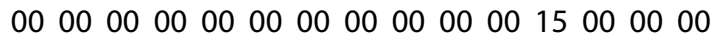 & 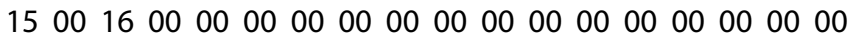 \\
\hline & 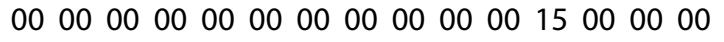 & 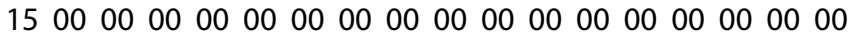 \\
\hline & 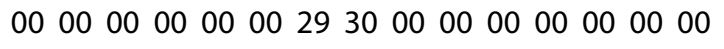 & 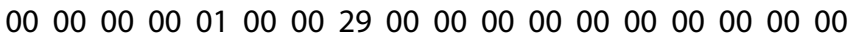 \\
\hline & $\begin{array}{lllllllllllll}00 & 00 & 00 & 00 & 29 & 30 & 00 & 00 & 00 & 00 & 00 & 00 & 00\end{array}$ & 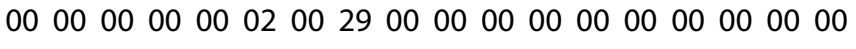 \\
\hline & $\begin{array}{llllllllllllll}00 & 00 & 00 & 00 & 00 & 30 & 00 & 00 & 00 & 00 & 00 & 00 & 00 & 00\end{array}$ & $\begin{array}{llllllllllllllllll}00 & 00 & 00 & 00 & 00 & 00 & 29 & 00 & 00 & 00 & 00 & 00 & 00 & 00 & 00 & 00 & 00\end{array}$ \\
\hline & 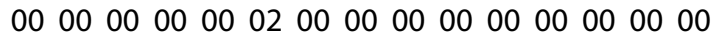 & 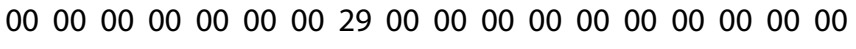 \\
\hline & 000000000002303000000006060616 & 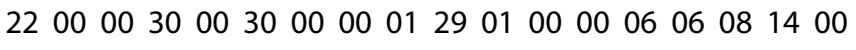 \\
\hline & 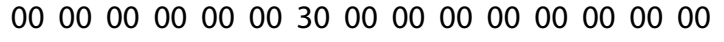 & 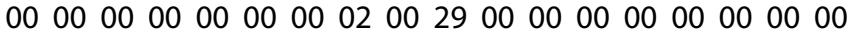 \\
\hline & $\begin{array}{lllllllllllllll}00 & 00 & 00 & 00 & 00 & 30 & 00 & 00 & 00 & 00 & 00 & 00 & 00 & 00\end{array}$ & 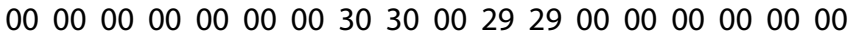 \\
\hline & 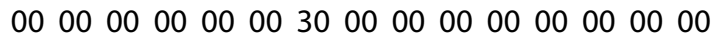 & 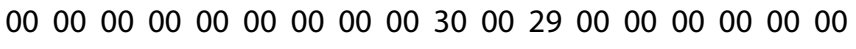 \\
\hline & 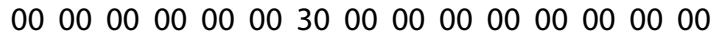 & 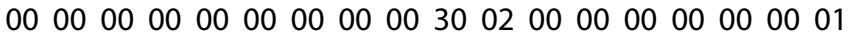 \\
\hline & $\begin{array}{llllllllllllll}00 & 00 & 00 & 00 & 00 & 00 & 27 & 00 & 00 & 28 & 00 & 00 & 00 & 00\end{array}$ & $\begin{array}{llllllllllllllllll}00 & 00 & 00 & 00 & 00 & 00 & 00 & 00 & 00 & 00 & 00 & 00 & 00 & 00 & 00 & 00 & 00\end{array}$ \\
\hline & 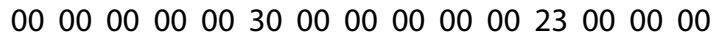 & 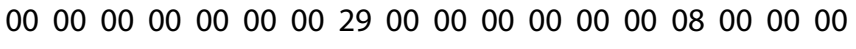 \\
\hline & 000000000030000000000023000000 & 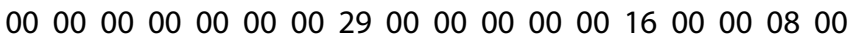 \\
\hline & 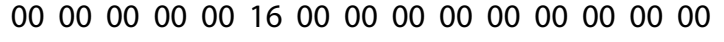 & 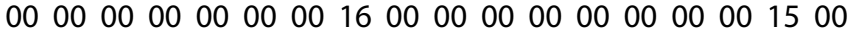 \\
\hline & $\begin{array}{llllllllllllll}00 & 00 & 00 & 00 & 22 & 00 & 00 & 00 & 00 & 00 & 00 & 00 & 00 & 00\end{array}$ & $\begin{array}{llllllllllllllllll}00 & 00 & 00 & 00 & 00 & 00 & 00 & 21 & 00 & 00 & 00 & 00 & 00 & 00 & 16 & 23 & 00 & 00\end{array}$ \\
\hline & 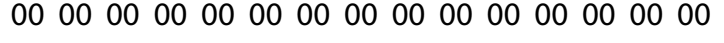 & 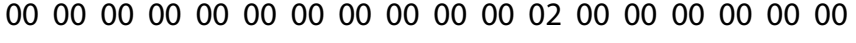 \\
\hline
\end{tabular}

11100000000000000000000000000000007 010011000000000000000000000000000 221000010000000000000000000000000 000001000000000000000000000000000 000001000000000000000010000000000 000001000000000000000010000000000

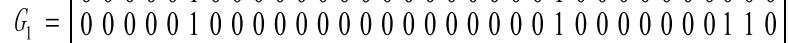
000000210000000000012020000000000 000000000000000000000011100000000 000000000000000000000000111000000 000000000000000000000000001000001 000000000000000000010100000000000000000

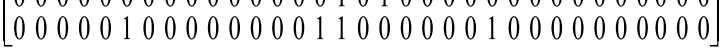




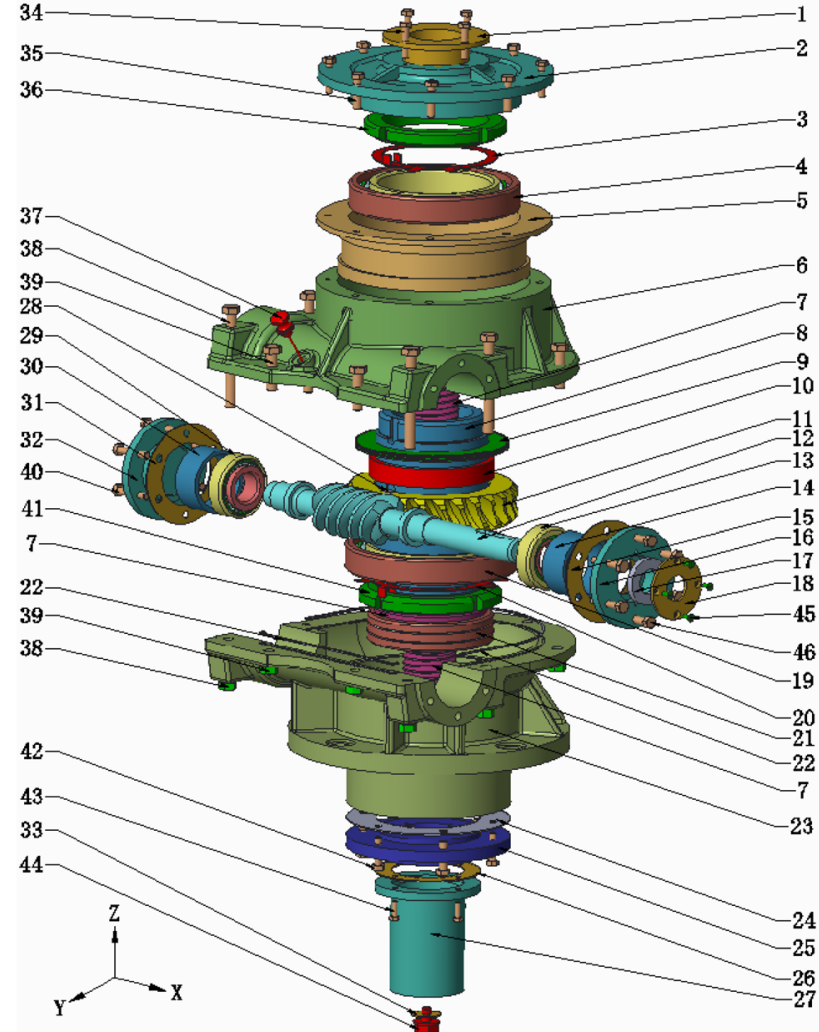

Fig. 5 Explosion view of screw lifting mechanism

Some parameters are set as follows:

The number of manipulators $I=3$; population size $p o p=100$, the number of frog subgroup $q_{s p}=10$; the iteration number of frog subgroup $l c=20$; the iteration number of population $I p=100$;

We first conduct an experimental analysis of the parameter $s_{\max }$. Comparing the different values of 6 groups, each group runs 10 times, and the distribution of 10 solutions obtained by different $s_{\max }$ are shown in Fig. 6.

It can be seen from Fig. 6 that the quality of the solution is poor when $s_{\max }=5$, because $s_{\max }$ is too small, so that the frog individual moves slowly during the evolution process. It always moves around itself and reduces the global search ability of the algorithm. It is easy to fall into local search. When $s_{\max } \geq 25$, the stability of the solution is poor. This is because $s_{\max }$ is too large, which makes the individual jump too much in the evolutionary process, so that it easily leads to the algorithm not finding the global optimal solution. The optimal value is obtained when $s_{\max }$ between 10 and 20 , but the overall quality of the solution is better when $s_{\max }=15$. Therefore, it is more appropriate to set $s_{\max }=15$.
Table 2 Disassembly information of screw lifting mechanism

\begin{tabular}{|c|c|c|c|c|}
\hline Index & Component & Tool & Dir & $\mathrm{Bt} / \mathrm{s}$ \\
\hline 1 & Upper end cap & Handwork T0 & $+Z$ & 5 \\
\hline 2 & Upper cap & Handwork T0 & $+Z$ & 10 \\
\hline 3 & Fixative collar 1 & Special tool T1 & $+Z$ & 3 \\
\hline 4 & Axle bearing 1 & Hand hammer T2 & $+Z$ & 6 \\
\hline 5 & Bearing support & Handwork T0 & $+Z$ & 15 \\
\hline 6 & Epivalve & Handwork T0 & $+Z$ & 25 \\
\hline 7 & Ballscrew & Special tool T3 & $+Z$ & 20 \\
\hline 8 & Axle & Hand hammer T2 & $+Z$ & 20 \\
\hline 9 & Washer & Special tool T1 & $+Z$ & 5 \\
\hline 10 & Collar & Hand hammer $\mathrm{T} 2$ & $+Z$ & 8 \\
\hline 11 & Worm gear & Special tool T4 & $+Z$ & 10 \\
\hline 12 & Worm & Special tool T4 & $+Z$ & 12 \\
\hline 13 & Worm bearing 1 & Hand hammer $\mathrm{T} 2$ & $+Z$ & 4 \\
\hline 14 & Shield ring 1 & forceps T4 & $+X$ & 4 \\
\hline 15 & Gasket 1 & Special tool T1 & $+X$ & 3 \\
\hline 16 & Worm cap1 & Handwork T0 & $+X$ & 4 \\
\hline 17 & Small washer & Special tool T1 & $+X$ & 3 \\
\hline 18 & Small cap & forceps T4 & $+X$ & 2 \\
\hline 19 & Axle bearing 2 & Hand hammer T2 & $-Z$ & 12 \\
\hline 20 & Fixative collar 2 & Special tool T1 & $-Z$ & 3 \\
\hline 21 & Bottom bearing & Hand hammer $\mathrm{T} 2$ & $-Z$ & 8 \\
\hline 22 & Box washer & Special tool T1 & $+Z$ & 8 \\
\hline 23 & Box & Handwork T0 & $-Z$ & 0 \\
\hline 24 & Soffit gasket & Special tool T1 & $-Z$ & 3 \\
\hline 25 & Soffit cap & Handwork T0 & $-Z$ & 6 \\
\hline 26 & Worm sleeve gasket & Special tool T1 & $-Z$ & 3 \\
\hline 27 & Worm sleeve & Handwork T0 & $-Z$ & 4 \\
\hline 28 & Bond & forceps T4 & $+\mathrm{Y}$ & 2 \\
\hline 29 & Worm bearing 2 & Hand hammer $\mathrm{T} 2$ & $-X$ & 4 \\
\hline 30 & Shield ring 2 & forceps T4 & $-X$ & 4 \\
\hline 31 & Gasket 2 & Special tool T1 & $-X$ & 3 \\
\hline 32 & Worm cap 2 & Handwork T0 & $-X$ & 4 \\
\hline 33 & Oil hole gasket & Special tool T1 & $-Z$ & 2 \\
\hline 34 & Setscrew of upper end cap & Wrench T5 & $+Z$ & 8 \\
\hline 35 & Setscrew of end cap & Wrench T5 & $+Z$ & 16 \\
\hline 36 & Jam nut 1 & Special Wrench T6 & $+Z$ & 6 \\
\hline 37 & Dipstick setscrew & Wrench T5 & $+Z$ & 4 \\
\hline 38 & Epivalve setscrew 1 & Wrench T5 & $+Z$ & 8 \\
\hline 39 & Epivalve setscrew 2 & Wrench T5 & $+\mathrm{Z}$ & 10 \\
\hline 40 & Setscrew of worm cap 2 & Wrench T5 & $-X$ & 9 \\
\hline 41 & Jam nut 2 & Special Wrench T6 & $-Z$ & 4 \\
\hline 42 & Setscrew of bottom cap & Wrench T5 & $-Z$ & 9 \\
\hline 43 & Setscrew of worm sleeve & Wrench T5 & $-Z$ & 8 \\
\hline 44 & Circumflex screw of oil hole & Wrench T5 & $-Z$ & 3 \\
\hline 45 & Setscrew of worm cap 1 & Wrench T5 & $+\mathrm{X}$ & 4 \\
\hline 46 & Setscrew of small cap & Screwdriver T7 & $+\mathrm{X}$ & 9 \\
\hline
\end{tabular}




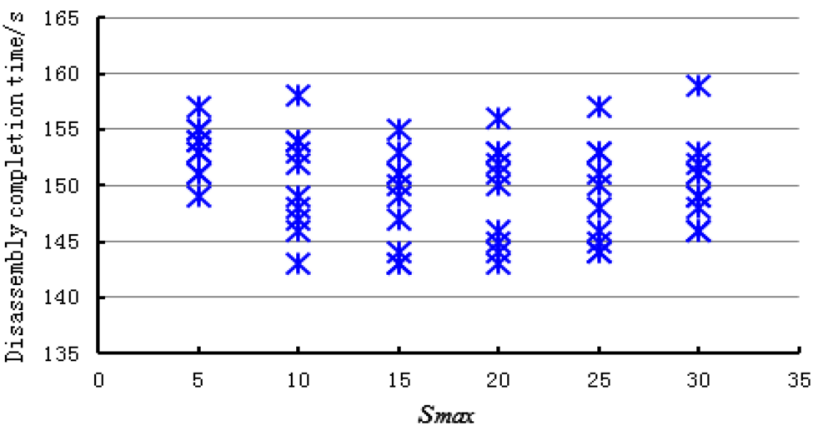

Fig. 6 Solution results under different leapfrog step

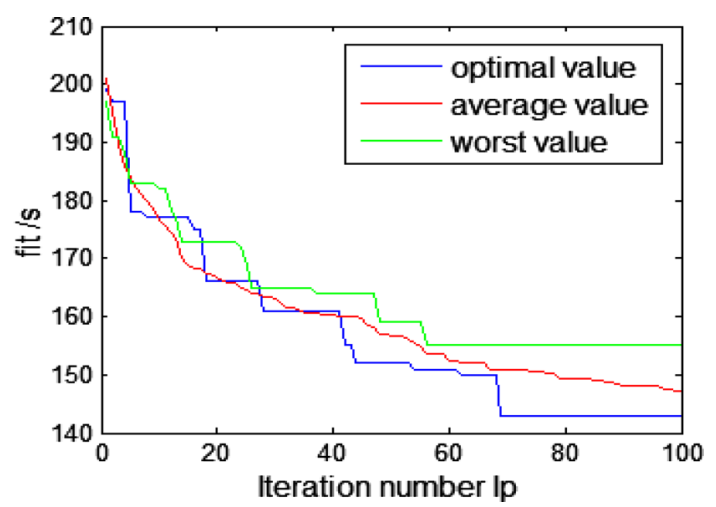

Fig. 7 Convergence curve at $s_{\max }=15$

When $s_{\max }=15$, the optimal value, average value and worst value convergence curve of test results are shown in Fig. 7.
It can be seen from Fig. 7 that as the number of iterations increases, all the convergence curves are steadily decreasing, and the average curve is approached to the optimal value curve. During the local evolutionary process, the worst individual of the frog groups evolved to the optimal individual of the sub-group or the optimal individual of the frog group and completed the location update. At the same time, in the function convergence process, there is no phenomenon that the individual function values are too concave or convex, which indicates that the proposed method has good stability and good order in the optimization process.

The optimal disassembly sequence is shown in Fig. 8 . The disassembly completion time is $143 \mathrm{~s}$.

\subsection{Case study 2}

In order to verify the feasibility and superiority of the proposed algorithm, the examples of different disassembly scales are selected and compared with genetic algorithm (GA) [9] and particle swarm optimization (PSO) [19]. The population size of the three algorithms pop $=100$, the number of iterations $I p=100$. Four different scales of disassembly examples (P1-P4) were selected. The examples $\mathrm{P} 1$ and $\mathrm{P} 4$ are the products to be disassembled shown in Figs. 1 and 5 respectively, the examples $\mathrm{P} 2$ and $\mathrm{P} 3$ are respectively in the literature $[38,39]$. Because the literature [39] does not give relevant disassembly information about the parts to be disassembly, therefore, the disassembly tool for all the parts is set to handwork T0, and other disassembly information is shown in Table 3.

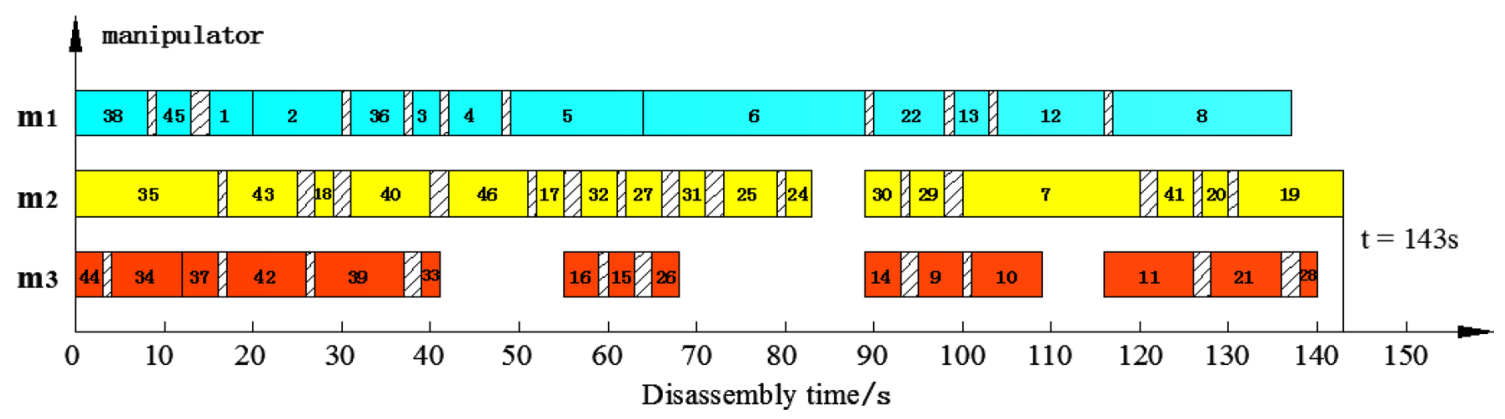

Fig. 8 Gantt chart of optimal disassembly sequence

Table 3 Disassembly information of $\mathrm{P} 3$

\begin{tabular}{lllllllllll}
\hline Index & 1 & 2 & 3 & 4 & 5 & 6 & 7 & 8 & 9 & 10 \\
\hline Bt/s & 1 & 2 & 1 & 2 & 2.5 & 3 & 2 & 4 & 1.5 & 2 \\
Dir & $+\mathrm{X}$ & $+\mathrm{X}$ & $+\mathrm{Y}$ & $+\mathrm{Y}$ & $+\mathrm{Y}$ & $+\mathrm{Y}$ & $+\mathrm{X}$ & $+\mathrm{X}$ & $-\mathrm{X}$ & $-\mathrm{X}$ \\
Index & 11 & 12 & 13 & 14 & 15 & 16 & 17 & 18 & 19 & 20 \\
Bt/s & 2.5 & 2.3 & 3 & 2 & 4 & 3 & 3 & 4 & 4 & 0.8 \\
Dir & $-\mathrm{X}$ & $-\mathrm{X}$ & $-\mathrm{X}$ & $-\mathrm{X}$ & $+\mathrm{Z}$ & $+\mathrm{Z}$ & $+\mathrm{X}$ & $+\mathrm{X}$ & $-\mathrm{Y}$ & $+\mathrm{X}$ \\
\hline
\end{tabular}


Table 4 basic information of 6 tests

\begin{tabular}{llll}
\hline Test Index & $\begin{array}{l}\text { Disassembly } \\
\text { instance }\end{array}$ & $\begin{array}{l}\text { The number of } \\
\text { components }\end{array}$ & $\begin{array}{l}\text { The number of } \\
\text { manipulators }\end{array}$ \\
\hline T1 & P1 & 10 & 2 \\
T2 & P2 & 23 & 2 \\
T3 & P3 & 39 & 2 \\
T4 & P3 & 39 & 3 \\
T5 & P4 & 46 & 2 \\
T6 & P4 & 46 & 3 \\
\hline
\end{tabular}

According to different disassembly scales and manipulators, a total of 6 tests (T1-T6) are set up. The basic information of each test is shown in Table 4. Each test runs for 10 times. The test results of each group are shown in Table 5. The convergence curve of optimal value under different algorithms is shown in Fig. 9.

In Table 5, the meanings of some parameters are as follows:

Et: the end time of disassembly, At: the average time of disassembly, Rt: the running time of the program.

It can be seen from the test result of $\mathrm{T} 1$ that when performing small-scale disassembly instance, all three algorithms can get the optimal or approximate optimal solution, and the running time and convergence speed of three algorithms are almost the same, but the overall quality of solution results of SFLA is better. By testing T2, the three algorithms show good stability when performing medium-scale disassembly. The optimal, worst, and average values of SFLA solution are better than GA and PSO, but SFLA converges slowly and the run time is a little longer. By testing T3 and T4, it can be seen that the SFLA solution is better than GA and PSO when the mediumlarge-scale disassembly is performed, and the convergence speed of SFLA is better than GA, but the advantage in terms of running time is not obvious. In addition, as the number of dismantling personnel increases, the disassembly completion time is significantly reduced. By testing $\mathrm{T} 5$ and T6, the optimal value, average value and worst values solved by SFLA are obviously better than GA and PSO when performing large-scale instances. SFLA has strong ability to jump out of local optimal solution, and the running time is slightly longer than GA and PSO.

According to the comparison and analysis of the test results of the different algorithms mentioned above, it can be known that the algorithm proposed in this paper shows excellent performance in solving disassembly instances of different sizes. Because the double-link encoding method proposed in this paper can effectively ensure the diversity of frog individuals. At the same time, the decoding method can achieve the full scheduling of disassembly personnel and disassembly tasks while ensuring the disassembly prior constraints. This paper adopts the idea of adjusting order based on the adjustment position to discretize the local evolution of frog individuals. A regulatory factor is expressed as a frog hop step size. By controlling the frog hop step size, the frog local search progress can be effectively controlled. This adjustment method can effectively avoid situations where the frog jump step is too large and no optimal solution can be found, or the frog jump step is too small and the search speed is too slow. In the process of global information interaction, mutation operators are added to effectively ensure the depth and diversity of information interaction.

In summary, the proposed algorithm can successfully solve disassembly examples of different scales, and the quality of the solution results is high. The optimization ability is especially excellent when solving large-scale disassembly examples.

\subsection{Case study 3}

In order to further testify the feasibility and superiority of the proposed algorithm, the algorithm in this paper is used to solve the examples in Ref. [38] and compared with the estimation of distribution algorithm (EODA) [38], branch and bound algorithm (BABA) [23], and genetic algorithm (GA) [39]. To ensure that the parameter settings are consistent with the literature [11], only the basic disassembly time of components is considered, and the population size $p o p=100$, the number of iterations $I p=100$. The test results are shown in Table 6.

As can be seen from Table 6, both ISFLA and EODA can obtain the optimal disassembly completion time, but the algorithm in this paper has an advantage in terms of running time. In addition, compared with the other two algorithms, the quality of the results solved by ISFLA is better. This is because the two other algorithms assume that each step of the task is performed simultaneously when solving parallel disassembly sequence planning, and do not fully consider the scheduling of the disassembly personnel and the disassembly tasks. Therefore, the algorithm proposed in this paper has certain advantages.

Through the above three case studies and analysis of the results, the method proposed in this paper can effectively solve disassembly cases of different sizes and types, and effectively solve the five problems raised in the literature review section of this article. The encoding and decoding methods proposed in this paper fully consider the cooperation and scheduling of multi-manipulator on the basis of apDSP. In addition, each step of the proposed method guarantees the feasibility of solutions. Therefore, the solution efficiency is higher and the results obtained when solving different cases are better than GA, PSO, and 
Table 5 Comparison of test results of three algorithms under different test conditions

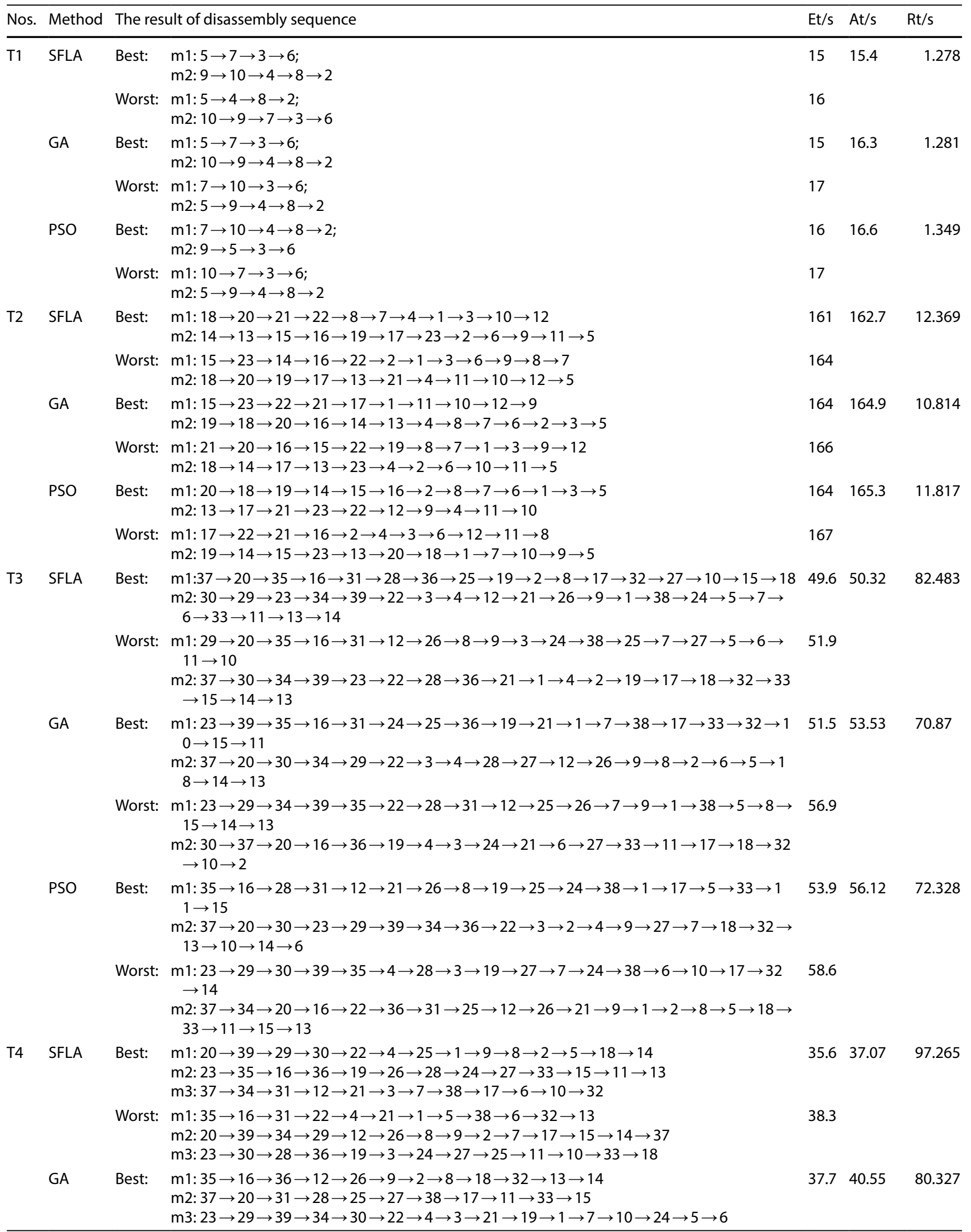


Table 5 (continued)

Nos. Method The result of disassembly sequence

Et/s At/s Rt/s

Worst: $\mathrm{m} 1: 37 \rightarrow 34 \rightarrow 36 \rightarrow 22 \rightarrow 12 \rightarrow 28 \rightarrow 25 \rightarrow 21 \rightarrow 8 \rightarrow 11 \rightarrow 33 \rightarrow 32$

43.6

$\mathrm{m} 2: 23 \rightarrow 39 \rightarrow 20 \rightarrow 31 \rightarrow 4 \rightarrow 26 \rightarrow 24 \rightarrow 38 \rightarrow 5 \rightarrow 2 \rightarrow 1 \rightarrow 18 \rightarrow 15$

$\mathrm{m} 3: 29 \rightarrow 30 \rightarrow 35 \rightarrow 16 \rightarrow 19 \rightarrow 3 \rightarrow 27 \rightarrow 9 \rightarrow 7 \rightarrow 6 \rightarrow 17 \rightarrow 10 \rightarrow 13 \rightarrow 14$

PSO Best: $\mathrm{m} 1: 20 \rightarrow 35 \rightarrow 16 \rightarrow 28 \rightarrow 24 \rightarrow 36 \rightarrow 19 \rightarrow 7 \rightarrow 9 \rightarrow 1 \rightarrow 18 \rightarrow 33$

$\mathrm{m} 2: 37 \rightarrow 23 \rightarrow 29 \rightarrow 30 \rightarrow 34 \rightarrow 39 \rightarrow 22 \rightarrow 3 \rightarrow 4 \rightarrow 25 \rightarrow 26 \rightarrow 21 \rightarrow 6 \rightarrow 10 \rightarrow 2 \rightarrow 14 \rightarrow 13$

$\rightarrow 15$

$\mathrm{m3:} 31 \rightarrow 12 \rightarrow 27 \rightarrow 11 \rightarrow 5 \rightarrow 8 \rightarrow 38 \rightarrow 17 \rightarrow 32$

Worst: $\mathrm{m} 1: 39 \rightarrow 37 \rightarrow 20 \rightarrow 35 \rightarrow 16 \rightarrow 28 \rightarrow 12 \rightarrow 27 \rightarrow 9 \rightarrow 11 \rightarrow 17 \rightarrow 14 \rightarrow 19$

$\mathrm{m} 2: 29 \rightarrow 31 \rightarrow 3 \rightarrow 24 \rightarrow 26 \rightarrow 7 \rightarrow 33 \rightarrow 38 \rightarrow 10 \rightarrow 32 \rightarrow 18 \rightarrow 21 \rightarrow 2$

$\mathrm{m} 3: 30 \rightarrow 23 \rightarrow 34 \rightarrow 22 \rightarrow 4 \rightarrow 25 \rightarrow 36 \rightarrow 8 \rightarrow 5 \rightarrow 15 \rightarrow 6 \rightarrow 13 \rightarrow 1$

T5 SFLA Best: $\mathrm{m} 1: 37 \rightarrow 35 \rightarrow 1 \rightarrow 46 \rightarrow 2 \rightarrow 36 \rightarrow 3 \rightarrow 4 \rightarrow 5 \rightarrow 6 \rightarrow 22 \rightarrow 9 \rightarrow 14 \rightarrow 10 \rightarrow 29 \rightarrow 41 \rightarrow 20 \rightarrow 1$ $9 \rightarrow 8$

$\mathrm{m} 2: 44 \rightarrow 42 \rightarrow 34 \rightarrow 43 \rightarrow 38 \rightarrow 45 \rightarrow 39 \rightarrow 40 \rightarrow 32 \rightarrow 31 \rightarrow 18 \rightarrow 17 \rightarrow 16 \rightarrow 15 \rightarrow 33 \rightarrow 27$

$\rightarrow 26 \rightarrow 25 \rightarrow 24 \rightarrow 30 \rightarrow 7 \rightarrow 13 \rightarrow 12 \rightarrow 11 \rightarrow 21 \rightarrow 28 \rightarrow 23$

Worst: $\mathrm{m} 1: 40 \rightarrow 38 \rightarrow 39 \rightarrow 43 \rightarrow 34 \rightarrow 1 \rightarrow 2 \rightarrow 36 \rightarrow 33 \rightarrow 16 \rightarrow 15 \rightarrow 27 \rightarrow 26 \rightarrow 25 \rightarrow 24 \rightarrow 22 \rightarrow 1$

$4 \rightarrow 13 \rightarrow 7 \rightarrow 41 \rightarrow 20 \rightarrow 8$

$\mathrm{m} 2: 45 \rightarrow 46 \rightarrow 42 \rightarrow 35 \rightarrow 44 \rightarrow 37 \rightarrow 18 \rightarrow 32 \rightarrow 17 \rightarrow 31 \rightarrow 3 \rightarrow 4 \rightarrow 5 \rightarrow 6 \rightarrow 30 \rightarrow 9 \rightarrow 29 \rightarrow$

$10 \rightarrow 12 \rightarrow 11 \rightarrow 28 \rightarrow 21 \rightarrow 19 \rightarrow 23$

GA $\quad$ Best: $\quad \mathrm{m} 1: 46 \rightarrow 40 \rightarrow 37 \rightarrow 35 \rightarrow 39 \rightarrow 44 \rightarrow 33 \rightarrow 27 \rightarrow 38 \rightarrow 3 \rightarrow 26 \rightarrow 5 \rightarrow 6 \rightarrow 7 \rightarrow 14 \rightarrow 10 \rightarrow 12$

$\rightarrow 11 \rightarrow 28 \rightarrow 21 \rightarrow 23$

$\mathrm{m} 2: 34 \rightarrow 42 \rightarrow 43 \rightarrow 45 \rightarrow 18 \rightarrow 1 \rightarrow 32 \rightarrow 31 \rightarrow 17 \rightarrow 2 \rightarrow 36 \rightarrow 4 \rightarrow 16 \rightarrow 25 \rightarrow 24 \rightarrow 15 \rightarrow 30$

$\rightarrow 22 \rightarrow 9 \rightarrow 29 \rightarrow 13 \rightarrow 41 \rightarrow 20 \rightarrow 19 \rightarrow 8$

Worst: $\mathrm{m} 1: 40 \rightarrow 44 \rightarrow 45 \rightarrow 38 \rightarrow 42 \rightarrow 46 \rightarrow 33 \rightarrow 32 \rightarrow 37 \rightarrow 36 \rightarrow 34 \rightarrow 5 \rightarrow 6 \rightarrow 30 \rightarrow 29 \rightarrow 14 \rightarrow 1$

$0 \rightarrow 24 \rightarrow 41 \rightarrow 19 \rightarrow 8$

$\mathrm{m} 2: 34 \rightarrow 43 \rightarrow 39 \rightarrow 35 \rightarrow 1 \rightarrow 2 \rightarrow 31 \rightarrow 18 \rightarrow 17 \rightarrow 27 \rightarrow 16 \rightarrow 26 \rightarrow 15 \rightarrow 25 \rightarrow 9 \rightarrow 22 \rightarrow 7$

$\rightarrow 13 \rightarrow 20 \rightarrow 12 \rightarrow 11 \rightarrow 28 \rightarrow 21 \rightarrow 23$

PSO Best: $\mathrm{m1}: 37 \rightarrow 44 \rightarrow 34 \rightarrow 45 \rightarrow 33 \rightarrow 46 \rightarrow 40 \rightarrow 32 \rightarrow 17 \rightarrow 43 \rightarrow 36 \rightarrow 31 \rightarrow 16 \rightarrow 27 \rightarrow 26 \rightarrow 25$

$\rightarrow 15 \rightarrow 14 \rightarrow 22 \rightarrow 12 \rightarrow 11 \rightarrow 21 \rightarrow 8$

$\mathrm{m} 2: 42 \rightarrow 38 \rightarrow 39 \rightarrow 18 \rightarrow 35 \rightarrow 1 \rightarrow 2 \rightarrow 3 \rightarrow 4 \rightarrow 5 \rightarrow 6 \rightarrow 24 \rightarrow 9 \rightarrow 30 \rightarrow 29 \rightarrow 13 \rightarrow 10 \rightarrow 7$

$\rightarrow 41 \rightarrow 20 \rightarrow 19 \rightarrow 28 \rightarrow 23$

Worst: $\mathrm{m} 1: 34 \rightarrow 44 \rightarrow 45 \rightarrow 43 \rightarrow 40 \rightarrow 18 \rightarrow 46 \rightarrow 1 \rightarrow 27 \rightarrow 2 \rightarrow 17 \rightarrow 25 \rightarrow 31 \rightarrow 15 \rightarrow 24 \rightarrow 5 \rightarrow 6$

$\rightarrow 7 \rightarrow 41 \rightarrow 14 \rightarrow 13 \rightarrow 12 \rightarrow 11 \rightarrow 28 \rightarrow 21$

$\mathrm{m} 2: 38 \rightarrow 39 \rightarrow 42 \rightarrow 35 \rightarrow 33 \rightarrow 32 \rightarrow 37 \rightarrow 26 \rightarrow 36 \rightarrow 16 \rightarrow 3 \rightarrow 4 \rightarrow 22 \rightarrow 30 \rightarrow 29 \rightarrow 9 \rightarrow 10$

$\rightarrow 20 \rightarrow 19 \rightarrow 8 \rightarrow 23$

T6 SFLA Best: $\mathrm{m} 1: 38 \rightarrow 45 \rightarrow 1 \rightarrow 2 \rightarrow 36 \rightarrow 3 \rightarrow 4 \rightarrow 5 \rightarrow 6 \rightarrow 22 \rightarrow 13 \rightarrow 12 \rightarrow 8$

$\mathrm{m} 2: 35 \rightarrow 43 \rightarrow 18 \rightarrow 40 \rightarrow 46 \rightarrow 17 \rightarrow 32 \rightarrow 27 \rightarrow 31 \rightarrow 25 \rightarrow 24 \rightarrow 30 \rightarrow 29 \rightarrow 7 \rightarrow 41 \rightarrow 20 \rightarrow$ 19

$\mathrm{m} 3: 44 \rightarrow 34 \rightarrow 37 \rightarrow 42 \rightarrow 39 \rightarrow 33 \rightarrow 16 \rightarrow 15 \rightarrow 26 \rightarrow 14 \rightarrow 9 \rightarrow 10 \rightarrow 11 \rightarrow 21 \rightarrow 28 \rightarrow 23$

Worst $\mathrm{m} 1: 38 \rightarrow 35 \rightarrow 1 \rightarrow 2 \rightarrow 31 \rightarrow 16 \rightarrow 30 \rightarrow 29 \rightarrow 14 \rightarrow 8 \rightarrow 21$

$\mathrm{m} 2: 45 \rightarrow 34 \rightarrow 44 \rightarrow 37 \rightarrow 33 \rightarrow 18 \rightarrow 32 \rightarrow 36 \rightarrow 3 \rightarrow 4 \rightarrow 5 \rightarrow 6 \rightarrow 7 \rightarrow 41 \rightarrow 20 \rightarrow 11 \rightarrow 28 \rightarrow$

23

$\mathrm{m} 3: 42 \rightarrow 40 \rightarrow 43 \rightarrow 46 \rightarrow 39 \rightarrow 27 \rightarrow 26 \rightarrow 17 \rightarrow 15 \rightarrow 25 \rightarrow 24 \rightarrow 9 \rightarrow 10 \rightarrow 13 \rightarrow 12 \rightarrow 22 \rightarrow$

19

GA $\quad$ Best: $\quad \mathrm{m} 1: 35 \rightarrow 39 \rightarrow 38 \rightarrow 32 \rightarrow 31 \rightarrow 18 \rightarrow 17 \rightarrow 4 \rightarrow 6 \rightarrow 24 \rightarrow 6 \rightarrow 7 \rightarrow 22 \rightarrow 41 \rightarrow 20 \rightarrow 11$

$\mathrm{m} 2: 43 \rightarrow 40 \rightarrow 42 \rightarrow 1 \rightarrow 2 \rightarrow 36 \rightarrow 5 \rightarrow 15 \rightarrow 14 \rightarrow 13 \rightarrow 29 \rightarrow 12 \rightarrow 19 \rightarrow 28$

$\mathrm{m} 3: 44 \rightarrow 33 \rightarrow 34 \rightarrow 37 \rightarrow 45 \rightarrow 46 \rightarrow 27 \rightarrow 26 \rightarrow 25 \rightarrow 3 \rightarrow 30 \rightarrow 9 \rightarrow 10 \rightarrow 8 \rightarrow 21 \rightarrow 23$

Worst: $\mathrm{m} 1: 35 \rightarrow 40 \rightarrow 46 \rightarrow 18 \rightarrow 32 \rightarrow 36 \rightarrow 3 \rightarrow 4 \rightarrow 5 \rightarrow 7 \rightarrow 41 \rightarrow 20 \rightarrow 19 \rightarrow 11 \rightarrow 28 \rightarrow 23$

$\mathrm{m} 2: 34 \rightarrow 39 \rightarrow 38 \rightarrow 37 \rightarrow 1 \rightarrow 2 \rightarrow 26 \rightarrow 24 \rightarrow 6 \rightarrow 14 \rightarrow 13 \rightarrow 22 \rightarrow 12 \rightarrow 8$

$\mathrm{m} 3: 43 \rightarrow 42 \rightarrow 44 \rightarrow 33 \rightarrow 45 \rightarrow 31 \rightarrow 17 \rightarrow 16 \rightarrow 27 \rightarrow 25 \rightarrow 15 \rightarrow 30 \rightarrow 9 \rightarrow 10 \rightarrow 29 \rightarrow 21$

$42.1 \quad 43.48 \quad 82.534$

45.8

Best: $\mathrm{m} 1: 18 \rightarrow 39 \rightarrow 38 \rightarrow 44 \rightarrow 33 \rightarrow 32 \rightarrow 31 \rightarrow 42 \rightarrow 37 \rightarrow 46 \rightarrow 26 \rightarrow 25 \rightarrow 15 \rightarrow 24 \rightarrow 11 \rightarrow 21$ $\rightarrow 8 \rightarrow 28 \rightarrow 23$

$\mathrm{m} 2: 45 \rightarrow 35 \rightarrow 27 \rightarrow 2 \rightarrow 36 \rightarrow 4 \rightarrow 5 \rightarrow 6 \rightarrow 9 \rightarrow 22 \rightarrow 14 \rightarrow 30 \rightarrow 12 \rightarrow 19$

$\mathrm{m} 3: 43 \rightarrow 34 \rightarrow 40 \rightarrow 17 \rightarrow 1 \rightarrow 3 \rightarrow 16 \rightarrow 7 \rightarrow 13 \rightarrow 29 \rightarrow 41 \rightarrow 20 \rightarrow 10$

Worst: $\mathrm{m1}: 44 \rightarrow 45 \rightarrow 39 \rightarrow 35 \rightarrow 38 \rightarrow 16 \rightarrow 33 \rightarrow 27 \rightarrow 26 \rightarrow 31 \rightarrow 4 \rightarrow 6 \rightarrow 22 \rightarrow 30 \rightarrow 29 \rightarrow 12 \rightarrow 1$

$1 \rightarrow 21 \rightarrow 28 \rightarrow 23$

$\mathrm{m} 2: 18 \rightarrow 37 \rightarrow 32 \rightarrow 17 \rightarrow 46 \rightarrow 36 \rightarrow 14 \rightarrow 9 \rightarrow 10 \rightarrow 24 \rightarrow 8$

$\mathrm{m} 3: 40 \rightarrow 42 \rightarrow 34 \rightarrow 43 \rightarrow 1 \rightarrow 2 \rightarrow 15 \rightarrow 3 \rightarrow 5 \rightarrow 13 \rightarrow 25 \rightarrow 7 \rightarrow 41 \rightarrow 20 \rightarrow 19$ 


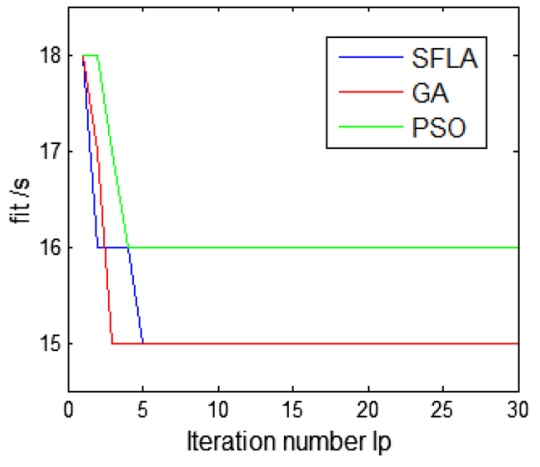

(a) Test T1

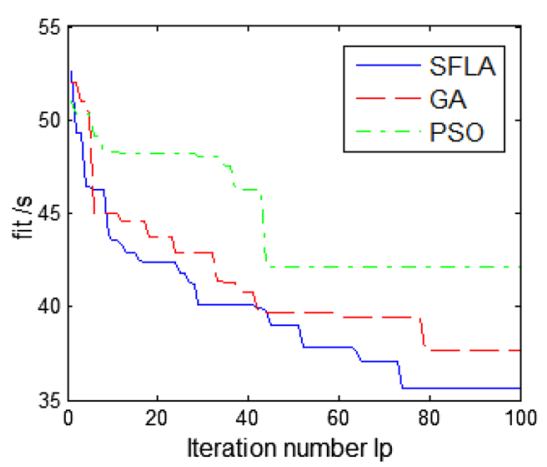

(d) Test T4

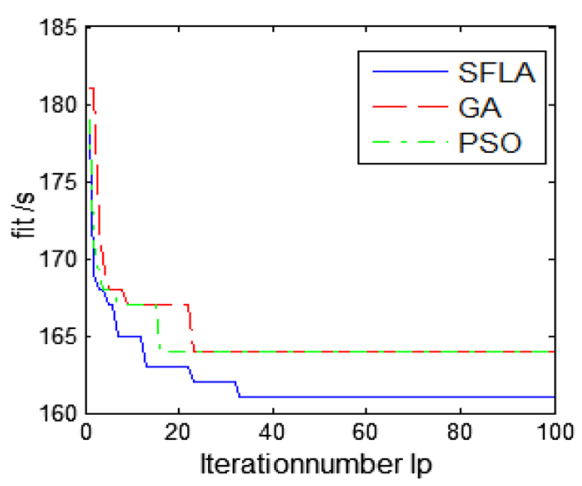

(b) Test T2

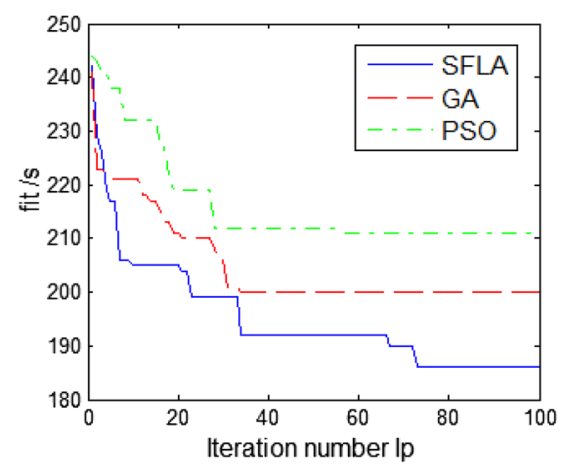

(c) Test T5

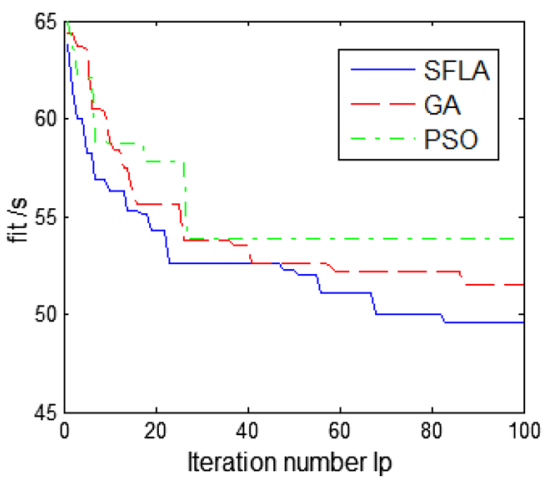

(c) Test T3

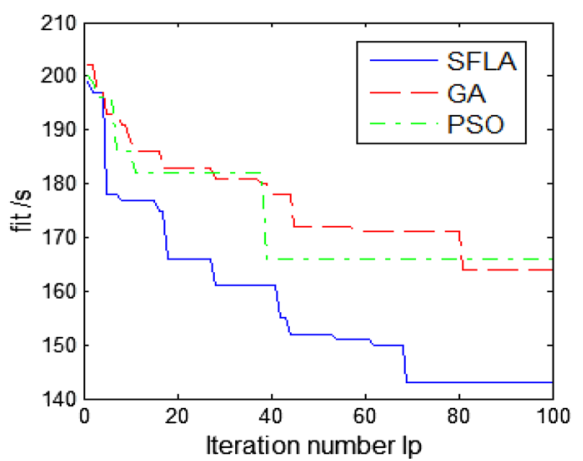

(f) Test T6

Fig. 9 Convergence curves of optimal solutions for three algorithms in different tests

Table 6 Comparison of test results

\begin{tabular}{lll}
\hline Algorithm & $\begin{array}{l}\text { Minimum disassembly com- } \\
\text { pletion time/s }\end{array}$ & $\begin{array}{l}\text { Running } \\
\text { time of } \\
\text { program }\end{array}$ \\
\hline ISFLA & 20.5 & 0.351 \\
EODA & 20.5 & 0.425 \\
BABA & 23 & 387.928 \\
GA & 23 & 0.233 \\
\hline
\end{tabular}

other algorithms. The disassembly model and solution method proposed in this article can express the feasible disassembly component of each disassembly personnel at any time. Based on the basic disassembly time of parts, the disassembly preparation time caused by the change of disassembly tools and directions is considered, so that the evaluation model is more realistic to reflect the actual disassembly process.

\section{Conclusions}

This paper proposes a method based on improved shuffled frog leaping algorithm to solve the problem of apDSP. We take the minimization of disassembly completion time as the optimization goal, and consider the disassembly preparation time due to the change of disassembly tool and direction. In order to fully express the disassembly constraints, the disassembly information model is constructed by using the fastener constraint matrix and the part constraint matrix, and the mathematical model of apDSP is constructed. To solve the problem of apDSP, we construct a double-link encoding structure and decoding operator, use the idea of adjustment order and doublepoint replacement to locally evolve the frog individual, and rebuild the formula of the frog position. In addition, the two-point mutation operator is used for the global information exchange to enhance the optimization ability of the algorithm. Finally, the effectiveness and superiority of the proposed algorithm are verified by a disassembly example of screw lifting mechanism and some cases of different disassembly scales. The experimental results show that the method proposed in this paper can solve various scales of disassembly examples, and the optimization 
ability is particularly excellent when performing largescale disassembly planning. The proposed algorithm has guiding significance for the actual disassembly work of complex assemblies.

Although the effectiveness and superiority of the proposed algorithm are verified by some disassembly cases, there are still some limitations exist. This work does not consider factors such as disassembly cost and profit in the process of disassembly evaluation. In addition, the proposed approach is mainly focus on complete disassembly, but a selective disassembly may present a better solution under some situations. All of these need to be considered into future efforts on advancing DSP.

Funding This study was funded by the National Natural Science Foundation of China (61272017) and the Science and Technology Innovation Commission of Shenzhen (JCYJ 20160510165328965).

\section{Compliance with ethical standards}

Conflict of interest The authors declare that they have no conflict of interest.

\section{References}

1. Meng K, Lou P, Peng X (2016) An improved co-evolutionary algorithm for green manufacturing by integration of recovery option selection and disassembly planning for end-of-life products. Int J Prod Res 54(18):5567-5593

2. Marco M, Claudio F, Michele G, Marco M (2018) Time-based disassembly method: how to assess the best disassembly sequence and time of target components in complex products. Int J Adv Manuf Technol 95:409-430

3. Li L, Huang H, Zhao F, Sutherland J (2017) An energy-saving method by balancing the load of operations for hydraulic press. IEEE/ASME Trans Mechatron 22(6):2673-2683

4. Qi L, Guang HZ, Zhong DX, Feng TC (2018) A selection methodology of key parts based on the characteristic of carbon emissions for low-carbon design. Int J Adv Manuf Technol 94:3359-3373

5. Li JR, Wang QH, Huang P (2012) An integrated disassembly constraint generation approach for product design evaluation. Int J Comput Integr Manuf 25(7):565-577

6. Dong TY, Zhang L, Tong RF (2006) A hierarchical approach to disassembly sequence planning for mechanical product. Int $J$ Adv Manuf Technol 30(5-6):507-520

7. Ren Y, Zhang C, Zhao F (2018) An asynchronous parallel disassembly planning based on genetic algorithm. Eur J Oper Res 269(2):647-660

8. Mohammad A, Ahmed ES, Elif K, Tarek M (2016) Disassembly sequencing using Tabu search. J Intell Robot Syst 82(1):69-79

9. Lambert A, Gupta S (2008) Methods for optimum and near optimum disassembly sequencing. Int J Prod Res 46(11):2845-2865

10. Maroua K, Moez T, Nizar A (2015) Disassembly sequence planning based on a genetic algorithm. J Mech Eng Sci 229(12):2281-2290
11. Soran P, Mozafar S (2019) Intelligent selective disassembly planning based on disassemblability characteristics of product components. Int J Adv Manuf Technol 104:1769-1783

12. Tseng $\mathrm{H}$, Chang $\mathrm{C}$, Lee $\mathrm{S}$, Huang $\mathrm{Y}$ (2018) A block-based genetic algorithm for disassembly sequence planning. Expert Syst Appl 96:492-505

13. Xu D, Jiao Q, Li C (2015) Project disassembly sequence planning based on adaptive genetic algorithm. Front Manuf Sci Meas Technol 11:372-375

14. Tseng $\mathrm{H}$, Chang $\mathrm{C}$, Lee $\mathrm{S}$, Huang $Y$ (2019) Hybrid bidirectional ant colony optimization (hybrid BACO): an algorithm for disassembly sequence planning. Eng Appl Artif Intell 83:45-56

15. Hu B, Feng Y, Hao Z, Tan J (2018) Sequence planning for selective disassembly aiming at reducing energy consumption using a constraints relation graph and improved ant colony optimization algorithm. Energies 11(8):1-18

16. Tseng YJ, Yu F, Huang F (2011) A green assembly sequence planning model with a closed-loop assembly and disassembly sequence planning using a particle swarm optimization method. Int J Adv Manuf Technol 57:1183-1197

17. Kheder M, Trigui M, Aifaoui N (2017) A disassembly sequence planning approach based on particle swarm optimization. In: Proceedings of 7 th conference on design and modeling of mechanical systems-III: LNME, pp 1017-1025

18. Tian G, Zhou M, Li P (2018) Disassembly sequence planning considering fuzzy component quality and varying operational cost. IEEE Trans Autom Sci Eng 15(2):748-760

19. Song S, Zhang W, Zhang L (2018) Product disassembly sequence planning based on improved artificial bee colony algorithm. China Mech Eng 27(17):2384-2390

20. Jiao Q, Xu D, Li C (2016) Product disassembly sequence planning based on flower pollination algorithm. Comput Integr Manuf Syst 22(12):2791-2799

21. Zhang XF, Zhang SY (2010) Product cooperative disassembly sequence planning based on branch-and-bound algorithm. Int J Adv Manuf Technol 51:1139-1147

22. Tian YY, Zhang XF, Xu JF (2018) Selective parallel disassembly sequence planning method for remanufacturing. J Comput Aid Des Comput Gr 30(3):531-539

23. Cai KJ, Zhang WM, Zhang MJ (2016) Disassembly sequence planning for multi-people simultaneous operation. Comput Integr Manuf Syst 22(12):2767-2777

24. Zhang XF, Yu G, Hu ZY (2014) Parallel disassembly sequence planning for complex products based on fuzzy-rough sets. Int J Adv Manuf Technol 72:231-239

25. Smith S, Huang PY (2015) A novel selective parallel disassembly planning method for green design. J Eng Des 26:283-301

26. Eusuff MM, Lansey KE (2003) Optimization of water distribution network design using the shuffled frog leaping algorithm. J Water Resour Plan Manag 129(3):210-225

27. Zhang Y, Gao XX, Wang YJ (2018) Solving the time optimal traveling salesman problem based on hybrid shuffled frog leaping algorithm-genetic algorithm. J Electron Inf Technol 40(2):363-370

28. Wu XJ, Wang JW, Huang GX (2015) Improved shuffled frog leaping algorithm for solving traveling salesman problems. J Chin Comput Syst 36(5):1078-1081

29. Feng YH, Wang GG, Gao XZ (2016) A novel hybrid cuckoo search algorithm with global harmony search for 0-1 knapsack problems. Int J Comput Intell Syst 9(6):1174-1190

30. Gao SQ, Xing YX, Xiao N (2018) Greedy frog leaping algorithm for 01 knapsack problem. Comput Sci 45(7):73-77

31. Meng HJ, Li YG (2015) An improved shuffled frog leaping algorithm for time-table problems. In: Proceedings of the 2015 international symposium on computers and informatics, vol 13, pp 991-997 
32. Pan QK, Wang L, Gao L, Li JQ (2011) An effective shuffled frogleaping algorithm for lot-streaming flow shop scheduling problem. Int J Adv Manuf Technol 52:699-713

33. Yang DJ, Lei $D$ (2018) A novel shuffled frog-leaping algorithm for FJSP with total energy consumption constraints. China Mech Eng 29(22):2682-2689

34. Xu Y, Wang L, Liu M, Wang SY (2013) An effective shuffled frog-leaping algorithm for hybrid flow-shop scheduling with multiprocessor tasks. Int J Adv Manuf Technol 68:1529-1537

35. Gonzalez B, Adenson DB (2006) Ascatter search approach to the optimum disassembly sequence problem. Comput Oper Res 33(6):1776-1793

36. Zhu Z, Xu Z, Shen W, Yang D (2018) Selective- disassembly sequence planning based on genetic-bat algorithm. J Zhejiang Univ (Eng Sci) 52(11):2120-2127
37. Xia K (2016) Research on the modeling and solution methods of disassembly sequence planning problem. Hua-zhong University of Science and Technology, Wuhan

38. Guo YQ, Zhang XF (2018) Parallel disassembly sequence planning based on estimation of distribution algorithm. J Mech Electr Eng 35:1023-1029

39. Zhang X, Wang YG, Wang $L$ (2015) Parallel disassembly sequence planning for complex products based on genetic algorithm. J Comput Aided Des Comput Gr 27(7):1327-1333

Publisher's Note Springer Nature remains neutral with regard to jurisdictional claims in published maps and institutional affiliations. 